Published in final edited form as:

ACS Infect Dis. 2016 March 10; 2(3): 207-220. doi:10.1021/acsinfecdis.5b00115.

\title{
Plantazolicin is an ultra-narrow spectrum antibiotic that targets the Bacillus anthracis membrane
}

\author{
Katie J. Molohon ${ }^{1,2, \#, ~ P a t r i c i a ~ M . ~ B l a i r ~}{ }^{3, \#, ~ S e o n g j i n ~ P a r k ~}{ }^{4}$, James R. Doroghazi ${ }^{2}$, Tucker \\ Maxson $^{3}$, Jeremy R. Hershfield ${ }^{5}$, Kristen M. Flatt ${ }^{6}$, Nathan E. Schroeder ${ }^{6}$, Taekjip Ha ${ }^{3,4,7}$, \\ and Douglas A. Mitchell ${ }^{1,2,3,{ }^{*}}$ \\ ${ }^{1}$ Department of Microbiology, University of Illinois at Urbana-Champaign, Urbana, IL 61801 \\ ${ }^{2}$ Carl R. Woese Institute for Genomic Biology, University of Illinois at Urbana-Champaign, Urbana, \\ IL 61801
}

${ }^{3}$ Department of Chemistry, University of Illinois at Urbana-Champaign, Urbana, IL 61801

${ }^{4}$ Department of Physics and Center for the Physics of Living Cells, University of Illinois at UrbanaChampaign, Urbana, IL 61801

${ }^{5}$ Bacteriology Division, United States Army Medical Research Institute of Infectious Diseases, Fort Detrick, MD 21702

${ }^{6}$ Department of Crop Sciences, University of Illinois at Urbana-Champaign, Urbana, IL 61801

${ }^{7}$ Howard Hughes Medical Institute, Chevy Chase, MD 20815

\begin{abstract}
Plantazolicin (PZN) is a ribosomally synthesized and post-translationally modified natural product from Bacillus methylotrophicus FZB42 and Bacillus pumilus. Extensive tailoring to twelve of the fourteen amino acid residues in the mature natural product endows PZN with not only a rigid, polyheterocyclic structure, but also antibacterial activity. Here we report a remarkably discriminatory activity of PZN toward Bacillus anthracis, which rivals a previously-described gamma $(\gamma)$ phage lysis assay in distinguishing $B$. anthracis from other members of the Bacillus cereus group. We evaluate the underlying cause of this selective activity by measuring the RNA expression profile of PZN-treated $B$. anthracis, which revealed significant upregulation of genes within the cell envelope stress response. PZN depolarizes the $B$. anthracis membrane like other cell envelope-acting compounds but uniquely localizes to distinct foci within the envelope.
\end{abstract}

\footnotetext{
*Corresponding Author (D.A.M.): douglasm@illinois.edu, Mailing address: 1206 West Gregory Drive, Carl R. Woese Institute for Genomic Biology, Room 3105, University of Illinois, Urbana, IL 61801 USA.

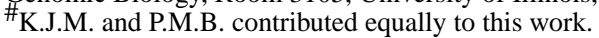

Author contributions

Conceived and designed the experiments: K.J.M., P.M.B., D.A.M. Performed the experiments: K.J.M., P.M.B., J.R.D., S.P., J.R.H., K.M.F. Analyzed the data: K.J.M., P.M.B., J.R.D., D.A.M., Contributed reagents/materials/analysis tools: T.M., N.E.S., T.H. Wrote the paper: K.J.M., P.M.B., D.A.M. All authors reviewed and approved the paper.

Notes

The authors declare no competing financial interest.

Supporting Information

Supporting information contains additional methods, supplementary tables, supplementary figures, and movies. This information is available free of charge via the Internet at http://pubs.acs.org/.
} 
Selection and whole-genome sequencing of PZN-resistant mutants of $B$. anthracis implicate a relationship between the action of PZN and cardiolipin (CL) within the membrane. Exogenous CL increases the potency of PZN in wild type $B$. anthracis and promotes the incorporation of fluorescently tagged PZN in the cell envelope. We propose that PZN localizes to and exacerbates structurally compromised regions of the bacterial membrane, which ultimately results in cell lysis.

\section{Keywords}

Ribosomally synthesized and post-translationally modified natural product; antibiotic; mode of action; pathogen specific antibiotic; membrane depolarization; Bacillus anthracis; anthrax; thiazole; oxazole

The current practice of employing broad-spectrum antibiotics to treat bacterial infections contributes to the rise of antibiotic resistance. ${ }^{(1)}$ As a countermeasure, species-selective and narrow-spectrum antibacterial compounds are garnering increased attention in the medical community for their potential as therapeutics and/or diagnostics. ${ }^{(2,3)}$ Plantazolicin (PZN) is a polyheterocyclic, linear compound of the ribosomally synthesized and post-translationally modified peptide (RiPP) natural product family with narrow-spectrum antibiotic activity (Figure S1). ${ }^{(4)}$ More specifically, PZN is a member of the thiazole/oxazole-modified microcins (TOMMs), a recently grouped and rapidly expanding RiPP class with $\sim 1,500$ identified gene clusters. ${ }^{(5,6)}$ Previously, PZN was described as an antibiotic compound that inhibits Gram-positive organisms closely related to its producing organism, Bacillus amyloliquefaciens FZB42(4) (this organism has recently been taxonomically reclassified as Bacillus methylotriphicus FZB42). ${ }^{(7)}$ In 2011, by screening a small panel of microorganisms, we described PZN as having potent activity towards Bacillus anthracis, but not other Gram-positive pathogens. ${ }^{(8)}$ Several additional PZN-like gene clusters have been identified in six distinct bacterial genera (from three phyla) through genome mining, but experimental data on antibiotic specificity has so far been limited to PZN. ${ }^{(4,8)}$ Although PZN has been the subject of total synthesis, ${ }^{(9-11)}$ heterologous expression, ${ }^{(12)}$ and enzymological studies, ${ }^{(13-15)}$ insight into the mode of action (MOA) of PZN has not been reported in the eight years since the discovery of its biosynthetic gene cluster. ${ }^{(16)}$

$B$. anthracis, the causative agent of anthrax and a category A priority pathogen, is a Grampositive bacterium and a member of the $B$. cereus sensu lato group, which includes $B$. cereus, B. anthracis, B. thuringiensis, and B. mycoides. ${ }^{(17)}$ Microbiologists have debated whether these organisms should be considered as one species, given that some strains share greater than 99\% DNA sequence identity. Despite being grouped with other Bacillus species, $B$. anthracis harbors a number of distinguishing features compared to other members of the $B$. cereus group. Fully virulent $B$. anthracis contains two conserved plasmids, $\mathrm{pXO}$ and $\mathrm{pXO}$, which harbor the genes responsible for producing the anthrax toxin and poly-Dglutamic acid capsule, respectively. However, homologous plasmids are also found in certain B. cereus strains. ${ }^{(18)}$ Beyond characteristic plasmid content, B. anthracis, unlike other members of the $B$. cereus group, harbors a nonsense mutation in plcR (phospholipase $\mathrm{C}$ regulator), rendering $B$. anthracis non-motile and non-hemolytic. ${ }^{(18)}$ The unambiguous 
differentiation of $B$. anthracis from other nonpathogenic $B$. cereus species is critically important from a public health perspective, especially as it pertains to bioterrorism.

Other defining features of $B$. anthracis that may facilitate species selectivity are exterior to the cell wall. $B$. anthracis displays a two-dimensional protein lattice called the surface layer (Slayer). Decorated with surface-associated proteins in a csaB (cell surface attachment)dependent manner, ${ }^{(19)}$ the S-layer is non-covalently attached to the secondary cell wall polysaccharide (SCWP), ${ }^{(20)}$ which is covalently tethered to the peptidoglycan. The $B$. anthracis SCWP is structurally unique ${ }^{(21)}$ and serves as the binding site for gamma $(\gamma)$ phage ${ }^{(22,23)}$ and previously described $B$. anthracis typing antibodies. ${ }^{(24)} \gamma$ phage produce a peptidoglycan hydrolase, PlyG, which specifically recognizes the terminal galactoses of the B. anthracis SCWP, allowing efficient digestion of the cell wall. ${ }^{(22)}$ Similarly, typing methods using monoclonal antibodies to the SCWP also exploit differences in the terminal sugar unit. However, there exist atypical $B$. anthracis strains that would constitute falsenegatives in any diagnostic assay based on these methods. ${ }^{(24,25)}$ Wip1, another $B$. anthracisspecific phage, is even more selective than $\gamma$ phage, yet certain $B$. cereus strains remain sensitive. ${ }^{(26)}$ Thus, the species specificity of PZN is intriguing not only from a MOA standpoint, but also as a rapid means to discriminate $B$. anthracis from other $B$. cereus sensu lato members.

Here we describe PZN as a remarkably selective small molecule antibiotic towards $B$. anthracis. The specificity of PZN was first examined by gene expression profiling, which yielded an expression signature distinct from broader spectrum antibiotics. We have identified and characterized a set of resistant mutants and evaluated their role in PZN resistance, which led us to further investigate the bacterial membrane as the most probable target of PZN. Using fluorescence-based approaches, we confirmed that PZN localizes to the cell envelope in a species-selective manner. PZN binding was associated with rapid and potent membrane depolarization. Taken together with the observation that PZN interacts synergistically with the negatively charged phospholipid, cardiolipin (CL), we propose that PZN localizes to and aggravates transient weaknesses present in the B. anthracis cell membrane.

\section{Results and Discussion}

$B$. anthracis, the causative agent of anthrax, can often be mistaken for other members of the B. cereus group. As a bacterium with history of use in bioterrorism, there is an urgent homeland security need for highly accurate and rapid identification of $B$. anthracis. We therefore set out to characterize and understand the selectivity of PZN.

\section{Defining the species selectivity of PZN}

PZN was originally described as a Gram-positive antibiotic, inhibiting the growth of $B$. subtilis, B. cereus, and B. megaterium. ${ }^{(4)}$ It is important to note, however, that the spot on lawn assay employed to reach this conclusion used $1 \mathrm{mg}$ of purified PZN per spot. We set out to obtain the minimum inhibiting concentration (MIC) of PZN by using a microbroth dilution assay. As expected, the activity of PZN was revealed to be considerably more selective, in that antibacterial activity was only detected toward $B$. anthracis upon screening 
a small panel of human pathogens. ${ }^{(8)}$ We continued to define this unusually narrow spectrum of activity by screening a larger panel of strains with varying degrees of genetic similarity (Table S1). PZN was found to be selective for vegetative $B$. anthracis, including fully virulent biosafety level 3 strains, with MICs between $1-16 \mu \mathrm{g} / \mathrm{mL}(0.75-12 \mu \mathrm{M})$. Endospores, the dormant phase of the $B$. anthracis life cycle, were resistant to PZN until germination was initiated (Table S2). By microbroth dilution, B. subtilis and B. cereus were not susceptible to PZN at concentrations up to $64 \mu \mathrm{g} / \mathrm{mL}$, which contrasts with the previous spot on lawn assay. ${ }^{(4)}$

To further investigate the selectivity of PZN towards B. anthracis, we conducted a head-tohead comparison using the $\gamma$ phage assay. Prior to modern genomic methods, $\gamma$ phage sensitivity and other phenotype testing were popular methods for identifying $B$. anthracis. ${ }^{(25)}$ Notwithstanding the reported $96 \%$ positive accuracy, non- $B$. anthracis strains that are sensitive to $\gamma$ phage and true $B$. anthracis strains that are insensitive have been reported. ${ }^{(25,27,28)}$ We obtained a panel of atypical B. cereus strains that are sensitive to $\gamma$ phage and tested them for PZN susceptibility (Table 1). B. cereus strains that generated a false positive in the $\gamma$ phage assay were not susceptible to PZN. ${ }^{(26,28,29)}$

To further define the attributes giving rise to the species selectivity of PZN, we procured various bacterial strains that address key differences between $B$. anthracis and $B$. cereus. plcR, encoding the phospholipase $\mathrm{C}$ regulator, is nonfunctional in $B$. anthracis but is intact in B. cereus. ${ }^{(18)}$ Deletion of plcR in B. cereus did not increase its susceptibility to PZN (Table $\mathrm{S} 1)$. Additionally, sortase-deficient strains of $B$. anthracis, which lack the ability to anchor various proteins to the cell wall, remain susceptible to PZN. ${ }^{(30)}$ The activity of PZN was similarly not dependent on the presence or composition of the $B$. anthracis S-layer, as strains deficient in Slayer assembly or decoration, namely those harboring mutations in $c s a B$, sap, and eag, are equally susceptible to PZN. ${ }^{(19)}$ We further confirmed that susceptibility to PZN is plasmid-independent given that a plasmid-deficient strain (LLNL A0517-1) and strains with both plasmids retained sensitivity (Table S1; Figure S2). Wip1 phage susceptibility and antibody typing have also been used to distinguish $B$. cereus sensu lato strains, but also have known exceptions to their specificity for $B$. anthracis. ${ }^{(24,26)}$ We obtained a "false-positive for B. anthracis" strain for each marker: B. cereus CDC32805 for Wip1 and B. cereus ATCC 7064 for antibody typing. We again observed no measurable PZN susceptibility for either strain (Table S1).

After extensive susceptibility testing, the only notable exception to the $B$. anthracis selectivity of PZN was B. cereus G9241 (MIC of $8 \mu \mathrm{g} / \mathrm{mL}$ ). Strain G9241 encodes the genes for an anthrax-like toxin on its pBCXO1 plasmid, which is named for its homology to the $B$. anthracis pXO1 plasmid. ${ }^{(31)}$ Because G9241 is encapsulated and toxigenic, it causes an anthrax-like disease but is undetectable in the $\gamma$ phage assay. ${ }^{(27)}$ Thus, from a pathogen detection perspective, the action of PZN towards G9241 could be considered fortuitous if it were to be further developed as a rapid diagnostic. Together, these data not only highlight the species discrimination of PZN but also rule out $p l c R$-related effects, sortase-mediated proteins, the SCWP, the S-layer, and plasmid-borne entities as targets of PZN. 
The spectrum of PZN activity calls into question whether bacteria are the naturally intended target. The canonical PZN-producing strain, B. methylotrophicus FZB42 (formerly amyloliquefaciens), is a prolific producer of other natural products with antifungal and nematicidal activities. ${ }^{7,32)}$ Liu et al. described a nematicidal activity to PZN, derived from experiments showing that PZN-deficient FZB42 strains exhibit reduced nematicidal activity against Caenorhabditis elegans. ${ }^{(33)}$ Since these experiments employed crude cellular extracts, we evaluated purified PZN in a similar manner, embedding the compound in agar ("slow killing" assay) or providing PZN in a liquid suspension ("liquid fast killing" assay). PZN was found to be no more toxic to $C$. elegans than a vehicle control and is not nematicidal in its own right (Figure S3). Purified PZN was also not responsible for the antifungal activity of the native producer, leaving the ecological function of PZN unknown (Table S1).

After observing the specificity of PZN under one growth medium condition (Luria-Bertani broth, LB), we re-assessed specificity against a smaller but representative panel of strains in two additional growth media (Mueller-Hinton and brain-heart infusion broths, Table S3). All tested strains of $B$. anthracis remained equally susceptible, but unexpectedly, some Staphylococcus aureus strains were susceptible to PZN under alternative growth media (MICs from $8-32 \mu \mathrm{g} / \mathrm{mL}$ ). Only $S$. aureus showed media-dependent susceptibility to PZN; all other tested strains remained non-susceptible to PZN.

\section{Assessing potential macromolecules as the target of PZN}

In an attempt to identify the molecular target(s) of PZN by affinity-based purification, ${ }^{(34)}$ three PZN derivatives were prepared: $N$-terminal biotinylation, $C$-terminal biotinylation, and $C$-terminal modification functionalized with aziridinyl and alkynyl groups for photoaffinity capture (Figure S4). Only the $C$-terminally modifications retained bioactivity, albeit with $\sim 16$-fold reductions; therefore, these probes were utilized for affinity-based target identification. Despite numerous attempts, we were unable to identify interactions unique to PZN compared to the control (data not shown). Because affinity purification-based strategies to identify small molecule targets is most successful when the interaction is of high-affinity to a protein, ${ }^{(35)}$ we considered the possibility that PZN may interact with a non-protein macromolecule. We thus monitored the formation of the cell wall, fatty acids, and RNA (as well as protein) using radiolabeled, biosynthetic precursors in the presence of PZN. Similar to daptomycin and the nisin-like lanthipeptide Pep5, PZN extensively disrupted macromolecular biosynthesis (Figure S5). ${ }^{(36,37)}$ Interestingly, and in contrast to vancomycin and daptomycin, PZN did not significantly block cell wall biosynthesis on the timescale of the experiment.

\section{The gene expression signature of PZN}

Sub-lethal antibiotic treatment stimulates rapid transcriptional responses in bacteria and the induced/repressed genes may be indicative of MOA. ${ }^{(38)}$ We thus performed RNA-Seq to evaluate the transcriptional response of $B$. anthracis following exposure to $0.25 \mu \mathrm{g} / \mathrm{mL}(0.25$ $\times$ MIC) PZN for 10 min. ${ }^{(39)} \mathrm{A}$ total of 74 genes were differentially regulated, including 63 upregulated and 11 downregulated genes, with an adjusted false discovery rate (q-value) of 0.01 (Figure 1; Table S4). The expression of a subset of these genes was validated by qRT- 
PCR (Tables S5-S6). Fourteen of the upregulated genes were transporter subunits, a common stress response upon antibiotic treatment. ${ }^{(40)}$ Conversely, PZN treatment led to the downregulation of genes associated with L-lactate metabolism, for which the implications remain unclear.

The most highly upregulated B. anthracis genes upon PZN treatment were bas 1344 and bas1345, which encode a hypothetical protein and a predicted member of the PspA/IM30 family, respectively (Table S6). These genes are homologous to the B. subtilis genes liaI and liaH (lipid II cycle interfering antibiotics), which are involved in the cell envelope stress response. Induction of these genes upon antibiotic treatment is well documented in $B$. subtilis, specifically to antibiotics interacting with lipid II in some capacity (e.g., nisin, vancomycin, and bacitracin). ${ }^{(41)}$ Induction of liaI and liaH is also seen in B. subtilis after daptomycin treatment, despite the lack of any known interaction between daptomycin and lipid II. ${ }^{(42)}$ PZN treatment also results in massive upregulation of bas5200 and bas5201, which are homologous to a $B$. subtilis thermosensor two-component system (desRK) that regulates the lipid desaturase, des. ${ }^{(43)}$ While bas5200 and bas5201 have yet to be experimentally interrogated, the response regulator and adjacent histidine kinase homologs are $62 / 89 \%$ and $38 / 71 \%$ similar/identical at the protein level, respectively. BLAST-P searches using BAS5200 and BAS5201 as the query sequences retrieve DesK and DesR as the highest similarity hits in B. subtilis; the reverse BLAST-P search (B. subtilis DesK and DesR query sequences) likewise retrieves BAS5200 and BAS5201 has the top hits in $B$. anthracis. Pending experimental validation of BAS5200-1 as the bona fide desRKTCS in $B$. anthracis, PZN would be to our knowledge the first compound known to alter the expression of these regulators, which is further suggestive of a unique MOA.

Recently, we reported on the synthesis of a PZN derivative, $\mathrm{Me}_{2}-\mathrm{Arg}-\mathrm{Az} 5$ (Figure S1). ${ }^{(15)}$ Chemically, $\mathrm{Me}_{2}-\mathrm{Arg}-\mathrm{Az}_{5}$ represents the $\mathrm{N}$-terminal half of PZN, but the activity spectrum of $\mathrm{Me}_{2}-\mathrm{Arg}-\mathrm{Az}_{5}$ is profoundly broader in LB and includes other Bacillus species as well as methicillin-resistant $S$. aureus (Table S7). Additionally, the later-described mutations in bas4114 that conferred resistance to PZN did not confer resistance to other antibiotics or to $\mathrm{Me}_{2}-\mathrm{Arg}-\mathrm{Az}_{5}$ (Tables S7-S8). To investigate their differing spectra of activity, we recorded the gene expression profile of $B$. anthracis treated with $\mathrm{Me}_{2}-\mathrm{Arg}_{-} \mathrm{Az}_{5}$ under otherwise identical conditions $(0.25 \times$ MIC, $10 \mathrm{~min})$ by RNA-Seq. The two compounds shared a minor portion of their expression profiles, but each profile was largely independent (Figure S6; Tables S9-S10). For example, sub-lethal $\mathrm{Me}_{2}-\mathrm{Arg}-\mathrm{Az}_{5}$ treatment also induced the $d_{e s R}$ two-component system, but expression of liaIH remained unchanged. Additionally, $\mathrm{Me}_{2}-$ Arg- $\mathrm{Az}_{5}$ failed to induce $B$. anthracis lysis, in contrast to PZN (Figure S7). A possible explanation for the observed differences between PZN and $\mathrm{Me}_{2}-\mathrm{Arg}-\mathrm{Az}_{5}$ is that the $C$ terminal portion of the molecule is responsible for the species selectivity of the mature molecule and the $N$-terminal portion harbors the antibiotic activity, although this remains to be more extensively investigated. The expression profiles of PZN and $\mathrm{Me}_{2}-\mathrm{Arg}-\mathrm{Az}_{5}$, together with strain susceptibility, suggest that $\mathrm{PZN}$ and $\mathrm{Me}_{2}-\mathrm{Arg}-\mathrm{Az} \mathrm{z}_{5}$ pursue independent, but possibly related, targets. Thus, while $\mathrm{Me}_{2}-\mathrm{Arg}-\mathrm{Az}_{5}$ is not useful as a mimic for the fulllength natural product, it represents one strategy to broaden the antibiotic spectrum of PZN. 


\section{PZN depolarizes the B. anthracis membrane}

The induction of liaIH and desRK by PZN suggests a potential relationship with components of the cell envelope. To determine if PZN treatment led to the loss of membrane potential, $3,3^{\prime}$-diethyloxacarbocyanine iodide $\left(\mathrm{DiOC}_{2}(3)\right)$ was used in a standard flow cytometry-based assay. ${ }^{(44)}$ We observed a dose-dependent decrease in membrane potential upon treating $B$. anthracis with PZN, even at concentrations 100-fold below the MIC. These data further suggest that PZN exerts its action by disrupting the integrity of the cell membrane (Figure 2). Destabilization of the bacterial cell membrane is a common MOA for antibiotics; for example, both nisin and daptomycin are known to disrupt membrane potential in Grampositive organisms. ${ }^{(45-47)}$ By co-treating $B$. anthracis with either nisin or daptomycin and PZN, the resulting isobolograms elicited strong synergistic activity with PZN (Figure 2), suggesting independent but cooperative activities. ${ }^{(48)}$

\section{Subcellular Localization of PZN}

With mounting evidence that PZN targets the cell membrane, we determined the subcellular localization of PZN by confocal microscopy. Antibiotics derivatized with fluorescent probes have previously been used to shed light on their MOAs. ${ }^{(49,50)}$ Localization of PZN was established by employing a Cy5-labeled PZN derivative (PZN-Cy5) (Figures S1, S8) that retained much of its anti- $B$. anthracis activity (MIC of $4 \mu \mathrm{g} / \mathrm{mL}, 2 \mu \mathrm{M}$ ). PZN-Cy5 localized to distinct $\sim 200 \mathrm{~nm}$ wide foci in B. anthracis Sterne (Figure 3a). To establish if PZN-Cy5 behaved in a manner identical to unlabeled PZN, we carried out a competition assay using an excess of unlabeled PZN applied to B. anthracis Sterne followed by addition of PZNCy5. Due to the extensive cell lysis elicited by PZN, we employed a later-described, spontaneous PZN-resistant mutant (PR06) for the competition assay. Just as in B. anthracis Sterne, PZN-Cy5 localized to distinct foci in B. anthracis PR06, which is consistent with its susceptibility, albeit at higher concentrations of PZN (Figure 3b). Importantly, PZN-Cy5 failed to label strain PR06 when an excess of unlabeled PZN was administered first, demonstrating that $\mathrm{PZN}$ and the PZN-Cy5 probe identically interact with $B$. anthracis (Figure 3c). Due to the photoswitching properties of Cy5, we were able to further investigate PZN-Cy5 localization using stochastic optical reconstruction microscopy (STORM, Figure 4). ${ }^{(51)}$ Using this super-resolution imaging technique, B. anthracis Sterne cells were again confirmed to accumulate PZN-Cy5 at the foci described above. These foci were clearly concentrated near the cellular surface, providing additional evidence that a component of the cell envelope as the target of PZN (Figure S9, Movies S1-S2). B. anthracis cells contain 16 \pm 2 foci per cell, each with a diameter of $181 \pm 7 \mathrm{~nm}$, as determined by analysis of 14 randomly chosen cells treated with PZN-Cy5 (Figure S9). The labeling pattern of PZN-Cy5 is strikingly different from that of BODIPY-vancomycin, which localizes strongly to bacterial septa where peptidoglycan synthesis is at a maximum. ${ }^{(52)}$ If PZN were acting on the cell wall, sites of active peptidoglycan synthesis or the entire cell wall would be labeled with PZN-Cy5. The non-septal, punctate labeling of PZN-Cy5 suggests that the target of PZN is neither nascent nor existing peptidoglycan, which is congruent with the observation that PZN did not block cell wall biosynthesis (Figure S5). PZN-Cy5 also does not appear to label non-susceptible Bacillus species (Figure S10). Some modestly labeling was found with B. cereus $\mathrm{G} 9241$, which aligns with a somewhat elevated MIC for PZN $(8 \mu \mathrm{g} / \mathrm{mL})$.

Combined with the evidence of PZN-Cy5 labeling PZN-resistant B. anthracis PR06 (Figure 
3), these data suggest that PZN binding to the cell envelope is necessary, but insufficient, for bacterial killing.

\section{Isolation and characterization of PZN-resistant mutants}

An orthogonal strategy for obtaining antibiotic MOA information involves the selection and mapping of resistance-conferring polymorphisms. ${ }^{(53)}$ The mutated gene(s) can either be involved directly in the MOA of the antibiotic or in a target-unrelated mechanism of immunity. We isolated PZN-resistant $B$. anthracis by growing the Sterne strain on agar plates containing PZN at $4 \times$ MIC. The resistance frequency was determined to be $2.3 \times 10^{-7}$, and the resulting mutants exhibited MICs that were $32 \mu \mathrm{g} / \mathrm{mL}$. Genomic DNA was isolated and sequenced for six independently-selected PZN-resistant strains (PR01 through PR06) and the parent Sterne strain. Comparison of PR01 through PR06 to the parent revealed that all six polymorphisms were confined to a 50-nucleotide section of a single gene, bas4114, which is annotated as an AcrR transcriptional repressor (Table S11). ${ }^{(54)}$ This particular AcrR protein is predicted to contain a single transmembrane domain near the $C$-terminus (Figure S11), which is precisely where the PZN-resistance conferring mutations were found, all resulting in premature stop codons. Directly downstream of bas4114 are two EmrE-type multidrug resistance efflux pumps, encoded by bas4115-4116. We hypothesized that as an AcrR-type transcriptional repressor, BAS4114 would negatively regulate bas4115-4116 and that mutations near the $C$-terminus of BAS4114 would result in regulator mislocalization/ dysfunction and derepression of the efflux pumps. This in turn would increase resistance to PZN. Multidrug resistant transporters have been shown to export membrane-associated antibiotics, ${ }^{(55,56)}$ a concept that is consistent with our data supporting the localization of PZN. One prediction is that BAS4115-6 actively efflux membrane-associated PZN, which lowers the steady-state membrane concentration of PZN. The MIC for PZN thus would increase with the increased expression of bas4115-4116. We employed RNA-Seq to compare the mRNA expression profiles of PR06 to the parent Sterne strain. This analysis revealed significant upregulation of bas4114-4116, as well as an unknown gene immediately downstream, bas4117 (Table S12). PR06 and the Sterne parent were equally susceptible to $\mathrm{Me}_{2}-\mathrm{Arg}-\mathrm{Az}_{5}$ (Table S7), again underscoring differences between PZN and $\mathrm{Me}_{2}-\mathrm{Arg}-\mathrm{Az}_{5}$. The susceptibility of PR06 to a panel of mechanistically-diverse antibiotics, including daptomycin, was also assessed (Table S8). The mutation present in PR06 did not confer cross resistance towards any other tested antibiotic. We then investigated if bas4114-4117 was constitutively overexpressed in non-susceptible B. cereus strains E33L and ATCC 4342, thereby conferring innate resistance to PZN. Using qRT-PCR, we could not identify constitutive overexpression of this locus in non-susceptible $B$. cereus strains compared to $B$. anthracis (data not shown). This suggests that the bas4114-4117 locus is not responsible for PZN resistance in non-susceptible $B$. cereus strains.

Frameshift mutations in the predicted transmembrane region of bas 4114 are clearly the favored route for generating PZN resistance in $B$. anthracis, as shown by the occurrence of multiple independent mutations within the same gene. In order to subvert this resistance mechanism, and to obtain more insightful information about the MOA of PZN, we deleted bas4114-4117 from the parental strain by homologous recombination (Figure S12). B. anthracis Sterne $\Delta$ bas 4114-4117 thus became the new parental strain for isolating second- 
generation PZN-resistant mutants, as the removal of bas4114-4117 rendered this strain as sensitive to PZN as wild type Sterne $(1 \mu \mathrm{g} / \mathrm{mL})$. This time, two routes were pursued for obtaining additional PZN-resistant strains. First, we selected spontaneous PZN-resistant mutants by challenging $\Delta$ bas4114-4117 with $4 \times$ MIC. Isolation of the spontaneous mutants resulted in a mutation frequency an order of magnitude lower than before $\left(1.3 \times 10^{-8}\right)$. Two independently-selected, modestly resistant strains (PR07 and PR08) were subjected to whole-genome sequencing, revealing single missense mutations within ftsE (Table 2). In Escherichia coli, FtsE is an ATP-binding protein that associates with its cognate permease, FtsX, together comprising an $\mathrm{ABC}$ transporter that functions during cell wall elongation and septum formation. ${ }^{(57)}$ The activity of FtsE/X in B. subtilis differs slightly, as it is responsible for initiating endospore formation via asymmetric septation. $\left.{ }^{(58)} 13\right)$. Akin to bas4114, spontaneous mutations in $f t s E$ alone cannot explain the species selectivity of PZN, as the amino acid sequence of $B$. anthracis FtsE is $100 \%$ identical to several non-susceptible $B$. cereus strains. Other B. cereus strains, even the PZN-susceptible G9241 strain, are 98-99\% identical to $B$. anthracis FtsE. While there may be an indirect relationship between PZN and FtsE/X, our data support a mode of action that does not involve a physical association with FtsE.

As a second strategy to obtain PZN-resistant mutants, we cultured $B$. anthracis Sterne $\triangle$ bas4114-4117 in the presence of a sub-lethal concentration of PZN. The concentration of PZN was gradually increased with the number of passages. ${ }^{(59)}$ We isolated genomic DNA from a $1^{\text {st }}$ passage strain (PR09-1, MIC $16 \mu \mathrm{g} / \mathrm{mL}$ ) in addition to two independent $4^{\text {th }}$ passage strains (PR09-4, PR10-4, MICs $₹ 64 \mu \mathrm{g} / \mathrm{mL}$ ) for whole genome sequencing. PR09-1 contained a missense mutation in bas1659, which encoded for a predicted CitB-like response regulator (Table 2). Downstream of bas 1659 are genes encoding a predicted histidine kinase (bas1660), ABC transporter subunits (bas1661-1663), and a cardiolipin (CL) synthase gene (bas1664). PR09-4 is a descendent of PR09-1, and as such, PR09-4 contained the same bas 1659 mutation as PR09-1 in addition to another missense mutation in bas1662 (the permease domain of the locally-encoded $\mathrm{ABC}$ transporter). PR10-4 contained a similar mutation series (bas1663, a second permease gene for what is presumably a trimeric $\mathrm{ABC}$ transporter) but had an additional mutation in bas 1842, which is implicated in petrobactin biosynthesis. ${ }^{(60)}$ Upon further inspection, we found that deletion of the petrobactin biosynthetic gene cluster did not decrease susceptibility to PZN, therefore the significance of this missense mutation remains unknown (Table S1).

\section{Exogenous cardiolipin increases sensitivity to PZN}

We hypothesized that the regulatory- and transport-related mutations upstream of the gene encoding CL synthase could alter CL concentrations and thus, CL may be implicated in the MOA for PZN. We first examined the effect of exogenous CL on the interaction of PZN with the $B$. anthracis cell membrane. $B$. anthracis cells were treated with PZN-Cy5 (1 nM, $0.001 \times$ MIC) in the presence and absence of exogenous CL (up to $100 \mu \mathrm{g} / \mathrm{mL}$ ). PZN-Cy5 treated cells were then analyzed by flow cytometry. The extent of PZN-Cy5 binding to $B$. anthracis was significantly increased when cells were co-treated with CL (Figure 5). This result is in contrast to that of daptomycin, which acts on the bacterial membrane but exhibits an antagonistic relationship with CL in Enterococci. ${ }^{(61,62)}$ As predicted, co-administration 
of CL did not increase the labeling efficiency of daptomycin-Cy5 on B. anthracis cells (Figures S1, 5, S13). ${ }^{(61)}$ Congruent with these data was the observation that CL potentiated the killing activity of PZN towards B. anthracis, but decreased daptomycin susceptibility 4fold. Indeed, the strongly synergistic behavior with CL enhanced the potency of PZN upwards of 16-fold while CL alone had no antibiotic activity at the concentrations tested (Figure 5).

\section{PZN colocalizes with cardiolipin and regions of increased fluidity}

The genetic and functional association with $\mathrm{CL}$ implicates the membrane as the most probable target for PZN. The lipid dye 10-N-nonyl acridine orange (NAO) approximates regions of the cell membrane enriched in CL. ${ }^{(63)}$ In B. subtilis and B. cereus, NAO organizes into distinct foci primarily at the septa and the poles (Figure S14), ${ }^{(64)}$ but it appears that in $B$. anthracis Sterne, NAO labels distinct foci throughout the entirety of the cell membrane (Figures 6 and S14). CL has the potential to dramatically alter membrane architecture and may contribute to the susceptibility of $B$. anthracis through punctate localization throughout the cell. We therefore treated $B$. anthracis cells with PZN-Cy5 and NAO to investigate if PZN localized to CL-rich regions in the cell membrane. There existed a clear but imperfect colocalization of the two dyes, suggesting a possible interaction with CL in the bacterial membrane (Figure 6). Thus, $B$. anthracis appears to have a unique distribution of CL that facilitates an interaction with PZN and leads to cell death, whereas CL localization within other species may not facilitate the lytic activity of PZN. Additionally, 1, $1^{\prime}$-didodecyl-3,3,3', $3^{\prime}$-tetramethylindocarbocyanine perchlorate, (DilC12(3)), is a dye reported to associate with regions of increased fluidity (RIF) within cell membranes of $B$. subtilis, and may also be indicative of CL localization. ${ }^{(65)}$ RIFs are transiently weakened regions within the bacterial membrane that affect lipid homeostasis and membrane fluidity. We observed colocalization of DilC12(3) and PZN-Cy5, consistent with PZN and CL co-associating with B. anthracis RIFs (Figure 6).

The proportion of CL in cell membranes has been reported to increase during growth in high osmolarity medium, especially for B. subtilis. ${ }^{(66)}$ We thus tested whether increasing the osmolarity of the $B$. subtilis medium (and thus the CL content) would induce susceptibility to PZN. When grown in standard LB supplemented with an additional $1.5 \mathrm{M} \mathrm{NaCl}(1.67 \mathrm{M}$ final), PZN was weakly growth-suppressive towards B. subtilis (Table 3). By measuring CL from total lipid extractions, CL levels did increase compared to standard growth in LB (Table 3). However, exogenous cardiolipin alone did not induce PZN susceptibility in $B$. subtilis or $B$. cereus (data not shown). Members of $B$. cereus sensu lato, including $B$. anthracis, are not as osmotolerant as $B$. subtilis; ${ }^{(67)}$ the maximum salinity these strains can tolerate in LB is $0.67 \mathrm{M}$ (standard LB supplemented with an additional $0.5 \mathrm{M} \mathrm{NaCl}$ ). When grown under high osmotic stress, both wild-type and PZN-resistant strains of $B$. anthracis display measurable susceptibility to PZN, although $B$. cereus strains remain resistant (Table 3). Unlike $B$. subtilis, cardiolipin content does not increase significantly when $B$. anthracis or $B$. cereus strains are grown in $0.5 \mathrm{M} \mathrm{NaCl}$, suggesting that the observed susceptibilities may be due to the harsh growth conditions rather than CL content. 
Cell survival under conditions of increased osmolarity is dependent on membrane fluctuations with an increase in unsaturated fatty acid composition. ${ }^{(66)}$ Increased CL levels are associated with high osmotic stress in B. subtilis, E. coli, Lactococcus lactis, and others. ${ }^{(66)}$ Furthermore, excess CL within the membrane results in increased fluidity and lipid bilayer deformation, as observed when $B$. subtilis is grown in a high osmolarity medium (Figure S15). ${ }^{(68)}$ By increasing the osmolarity of the growth medium, we can induce a modest increase in the potency of PZN towards $B$. anthracis (Table 3). The resultant increase in CL, together with the activation of the des two-component system, presumably shifts the lipid profile to a more fluid composition, which negatively affects membrane integrity. This phenotype is readily observed by confocal microscopy (Figure S15). ${ }^{(69)} \mathrm{We}$ hypothesize that PZN takes advantage of an already weakened $B$. anthracis membrane to elicit its selective antibacterial activity.

As stated previously, the stepwise-selected PZN-resistant strains accumulated mutations in genes upstream of one of five CL synthase genes (cls, bas 1664). We analyzed the transcriptional response within the bas 1664 locus, including the upstream regulators/ transporters and observed a dramatic increase in the expression of several genes in PR09-1 and PR09-4, including bas1664 itself and the nearby ABC transporter genes (bas1661-1663), but not the locally-encoded response regulator and histidine kinase genes (bas1659-1660, Table S13). In stark contrast, there was no differential expression of any tested gene in the PR10-4 strain. While these results may seem contradictory, PR10-4 did acquire an additional mutation in a gene responsible for the production of the siderophore petrobactin (Table 2). ${ }^{(70)}$ Given that it is well established that $B$. subtilis experience iron limitation when grown under high osmotic stress, ${ }^{(71,72)}$ it is possible that PR10-4 handles stress induced by PZN or high NaCl concentrations differently than PR09-1 and PR09-4. Further, CL levels do not necessarily correlate to the number of $c l s$ transcripts (and there are multiple cls genes), which is suggestive of other regulatory mechanisms to avoid overproduction of CL. ${ }^{(73)}$ With respect to PR09-1, PR09-4, and PR10-4, there is a complex relationship between CL content and PZN susceptibility. Based on our findings, we expect that in addition to $\mathrm{CL}$, other membrane-associated biomolecules may also contribute to the ability of PZN to destabilize $B$. anthracis cell membranes.

Due to the advance in genomics, $B$. anthracis, the causative agent of anthrax, can be distinguished from the other members of the $B$. cereus sensu lato group by whole genome sequencing, multilocus sequence typing, the presence of chromosomal lambdoid prophages, and the presence of a characteristic nonsense mutation in $p l c R^{(18,29)}$ An alternative approach to $B$. anthracis identification now includes susceptibility to PZN, which is a natural product exhibiting potent and selective bactericidal activity for $B$. anthracis under standard laboratory conditions. Our data demonstrate that the species selectivity of PZN is even more discriminating than that of the reputedly selective $\gamma$ phage. $(25,26,28,29)$ Additionally, PZN on its own does not contribute to B. methylotrophicus FZB42 antifungal or nematicidal activity. $B$. anthracis is nearly identical to other members of the $B$. cereus sensu lato family, and remarkably, strains of $B$. anthracis selected to interrogate key genetic differences retain their respective susceptibility to PZN. Gene expression analysis, together with confocal and super-resolution microscopy, reveals that PZN operates by a different MOA than previously described cell envelope-targeting antibiotics. Thus, we present a model for PZN activity 
wherein PZN takes advantage of a locally weakened cell membrane, whether due to RIFs, CL-dependent membrane deformation, or some combination thereof. PZN accumulates to such membrane defects, resulting in membrane depolarization and lysis of $B$. anthracis in a species-specific manner. The activity of PZN suggests an immediate homeland security application, where it could be developed into a rapid $B$. anthracis detection test.

\section{Methods}

\section{Strain and growth conditions}

All strain references are displayed in Table S1. All strains were grown in Luria-Bertani (LB) broth unless otherwise described $(10 \mathrm{~g} / \mathrm{L}$ tryptone, $5 \mathrm{~g} / \mathrm{L}$ yeast extract, $10 \mathrm{~g} / \mathrm{L}(0.34 \mathrm{M})$ $\mathrm{NaCl}$ ). Biosafety level 3 strains of $B$. anthracis were grown on Mueller-Hinton agar. Neisseria strains were grown in gonococcal medium base supplemented with Kellogg's I and II. ${ }^{(74)}$ Streptomyces endospores were isolated on mannitol soybean flour agar (20 g/L mannitol, $20 \mathrm{~g} / \mathrm{L}$ soybean flour, $1.5 \%$ agar) and used to determine PZN susceptibility in ISP2 (4 g/L yeast extract, $10 \mathrm{~g} / \mathrm{L}$ malt extract, $4 \mathrm{~g} / \mathrm{L}$ dextrose). Yeast strains were grown in YPD medium (10 g/L yeast extract, $20 \mathrm{~g} / \mathrm{L}$ peptone, $20 \mathrm{~g} / \mathrm{L}$ dextrose). C. elegans was cultured on nematode growth medium with $E$. coli OP50. Cultures were supplemented with $1.25 \mathrm{mM} \mathrm{CaCl}_{2}$ when assaying daptomycin susceptibility. In cases where increased osmolarity was desired, the $\mathrm{LB}$ was supplemented with additional $\mathrm{NaCl}$ (final concentration of $1.84 \mathrm{M})$.

\section{PZN Bioactivity}

$\mathrm{PZN}$ and $\mathrm{Me}_{2}-\mathrm{Arg}-\mathrm{Az}_{5}$ bioactivity was determined via microbroth dilution assay as described in the Clinical and Laboratory Standards Institute manual. ${ }^{(75)}$ The optical density $\left(\mathrm{OD}_{600}\right)$ of a stationary phase culture was adjusted to 0.01 and added to a microtiter plate containing serially diluted PZN. Wells were visually inspected for turbidity, and the minimum inhibitory concentration (MIC) was determined as the lowest compound concentration that incurred no growth after $16 \mathrm{~h}$. MICs were determined in LB unless growth conditions required an alternative medium (see above). The $S$. aureus mediadependent PZN susceptibility was analyzed using LB, Brain-Heart Infusion (BHI, Bacto), and Mueller-Hinton (BBL) broths. When indicated, cardiolipin (CL) was added to the medium at $100 \mu \mathrm{g} / \mathrm{mL}$.

A B. anthracis growth curve was generated using Tecan Infinite M200 Pro. B. anthracis Sterne 7702 cultures were grown in LB to stationary phase at $37^{\circ} \mathrm{C}$. Cultures were diluted to $\mathrm{OD}_{600}$ of 0.05 with fresh $\mathrm{LB}$ and allowed to recover to an $\mathrm{OD}_{600}$ of 0.35 . Cultures were aliquoted into 96-well plates containing PZN and incubated at $37^{\circ} \mathrm{C}$ with orbital shaking. $\mathrm{OD}_{600}$ was measured every $2 \mathrm{~min}$. Values were normalized to an initial $\mathrm{OD}_{600}$ of 0.35 and adjusted to a $1 \mathrm{~cm}$ path length. Error bars represent standard deviation of two independent experiments.

A growth curve in the presence of $\mathrm{Me}_{2}-\mathrm{Arg}-\mathrm{Az}_{5}$ was generated as described above with the following differences: $B$. anthracis Sterne 7702 and $S$. aureus USA300 cultures were grown in duplicate to $\mathrm{OD}_{600} 1.0$ and aliquoted into 96-well plates. Wells were treated with 1:1 
dilutions of $\mathrm{Me}_{2}-\mathrm{Arg}-\mathrm{Az}_{5}$ at a maximum concentration of $12 \mu \mathrm{M}$. The plate was incubated at $37{ }^{\circ} \mathrm{C}$ with orbital shaking and $\mathrm{OD}_{600}$ was measured every $2 \mathrm{~min}$. Values were normalized to an initial $\mathrm{OD}_{600}$ of 1.0 , adjusted to a $1 \mathrm{~cm}$ path length, and averaged at each time point.

\section{Gamma $(\gamma)$ phage sensitivity}

$\gamma$ phage were propagated as described previously ${ }^{(25)}$ using B. anthracis Sterne 7702 cells on brain heart infusion (BHI) agar plates, with no visible loss in infectivity. Phage infectivity was tested against a panel of $B$. cereus and $B$. anthracis strains using a serial dilution assay. Stationary phase cultures were adjusted to an $\mathrm{OD}_{600}$ of 0.1 and $100 \mu \mathrm{L}$ was plated on BHI plates. $5 \mu \mathrm{L}$ of phage stock $\left(2.6 \times 10^{8}\right.$ plaque forming units $/ \mathrm{mL}$ ) was serially diluted (2-fold) and spotted onto the plates and allowed to dry. After incubation at $37^{\circ} \mathrm{C}$ for $16 \mathrm{~h}$, plates were removed and visually inspected for plaques.

\section{RNA isolation and transcriptional profiling of PZN-treated Sterne cells}

For the compound-treated samples, independent $3 \mathrm{~mL}$ cultures of $B$. anthracis Sterne 7702 cells were grown to an $\mathrm{OD}_{600}$ of 0.4 , and $0.25 \times \mathrm{MIC}$ of $\mathrm{PZN}, 0.25 \times \mathrm{MIC} \mathrm{Me}_{2}-\mathrm{Arg}-\mathrm{Az}_{5},{ }^{(15)}$ or an equivalent volume of DMSO was added and allowed to incubate for $10 \mathrm{~min}$ at $37^{\circ} \mathrm{C}$. Together with resistant mutant PR06, RNA was isolated and prepared as described previously ${ }^{(4)}$. RNA-Seq libraries were created using the TruSeq Stranded RNA Sample Prep kit (Illumina, San Diego, CA) after rRNA depletion using the RiboZero Bacteria kit (Epicentre, Madison, WI). Sequencing was performed for $1 \times 100$ cycles on a HiSeq 2000 with Version 3 Chemistry. Transcriptomic data was processed with the Rockhopper version 1.30 pipeline ${ }^{(76)}$ using $B$. anthracis Sterne and B. anthracis Ames Ancestor plasmid pXO1 (NC_007322.2) as references. Default values (allowed mismatches 0.15, minimum seed length 0.33 , minimum expression of UTRs and ncRNAs 0.5 ) were used, with the exception that reverse complement reads were used for mapping. The RNA-Seq data discussed in this publication have been deposited in NCBI's Gene Expression Omnibus and is accessible through GEO Series accession number GSE73343 (http://www.ncbi.nlm.nih.gov/geo/query/ acc.cgi?acc $=$ GSE73343).

\section{Membrane depolarization}

Three independent stationary phase cultures of $B$. anthracis Sterne 7702 were used to inoculate fresh $\mathrm{LB}$ and grown to $\mathrm{OD}_{600} 0.5$ at $37^{\circ} \mathrm{C}$ with shaking. Aliquots $(10 \mu \mathrm{L})$ were diluted to $1 \mathrm{~mL}$ in PBS containing $0.1 \mu \mathrm{M} \mathrm{DiOC} 2$ (3) (3,3'-diethyloxacarbocyanine iodide) and compounds (DMSO - vehicle, $5 \mu \mathrm{M}$ carbonyl cyanide $m$-chlorophenyl hydrazone (CCCP), 3.0 $\mu \mathrm{M}$ daptomycin, $0.5 \mu \mathrm{M}$ PZN, $1.0 \mu \mathrm{M}$ PZN). Cells were mixed at $21^{\circ} \mathrm{C}$ for 30 min prior to analysis by flow cytometry (BD LSR II Flow Cytometry Analyzer). Voltages for fluorescein isothiocyanate (FITC) and propidium iodide (PI) fluorescence were set so that average counts per cell were between $10^{3}$ and $10^{4}$. Geometric means for fluorescence ratios were normalized to the control $\mathrm{DiOC}_{2}(3)$ samples.

\section{Confocal microscopy}

In general, cells were prepared by inoculating $5 \mathrm{~mL}$ LB with $200 \mu \mathrm{L}$ of a stationary phase culture. After growing to an $\mathrm{OD}_{600}$ of 0.5 at $37^{\circ} \mathrm{C}$ with shaking, $1 \mathrm{~mL}$ aliquots were 
centrifuged ( $3 \mathrm{~min}, 8000 \times g$ ), decanted, and resuspended in sterile PBS. Slides were prepared by mixing 1:1 (v/v) cell suspensions in PBS and liquefied low gelling temperature agarose (Sigma-Aldrich, $2 \% \mathrm{w} / \mathrm{v}$ in water). All microscopy images were obtained using a Zeiss LSM 700 Confocal microscope with a $63 \times / 1.4$ Oil DIC objective and processed using Zen 2012 software. Laser intensity and gain were kept at a minimum and held constant for all experiments. Linear contrast was equally applied during image processing. To localize PZN, B. anthracis Sterne 7702 was treated in PBS with $0.2 \mu \mathrm{M}$ PZN-Cy5 for $30 \mathrm{~min}$ at $22{ }^{\circ} \mathrm{C}$. After washing in PBS $(3 \times 500 \mu \mathrm{L})$, cells were resuspended in a final volume of 250 $\mu \mathrm{L}$ PBS. Competition experiments were performed using PR06 (PZN-resistant) in PBS treated with DMSO (vehicle) or $1 \mu \mathrm{M}$ PZN for $20 \mathrm{~min}$ at $22{ }^{\circ} \mathrm{C}$ before the addition of 0.05 $\mu \mathrm{M}$ PZN-Cy5. After $20 \mathrm{~min}$ at $21^{\circ} \mathrm{C}$, the cells were washed in PBS $(3 \times 500 \mu \mathrm{L})$ and resuspended in a final volume of $250 \mu \mathrm{L}$ PBS. Sterne underwent co-treatment in PBS with $0.2 \mu \mathrm{M}$ PZN-Cy 5 for $25 \mathrm{~min}$ before the addition of other fluorescent compounds. After 5 min additional treatment, cells were washed in PBS $(5 \times 500 \mu \mathrm{L})$ and resuspended in a final volume of $250 \mu \mathrm{L}$ PBS. Concentrations used: NAO (Sigma-Aldrich), $1 \mu \mathrm{M}$; Dil-C12, $1 \mu \mathrm{M}$; For CL experiments, cells were treated with EtOH (vehicle), $10 \mu \mathrm{g} / \mathrm{mL}$ CL, or $100 \mu \mathrm{g} / \mathrm{mL}$ $\mathrm{CL}$ in addition to $0.2 \mu \mathrm{M}$ PZN-Cy5 for $30 \mathrm{~min}$. For high osmolarity samples, cells were grown to stationary phase in standard LB and diluted into high osmolarity medium (an additional $1.5 \mathrm{M} \mathrm{NaCl}$ was added to $B$. subtilis cultures; $0.50 \mathrm{M}$ for $B$. anthracis and $B$. cereus).

\section{Super-resolution microscopy (STORM)}

Cells for 3D super-resolution microscopy were grown and treated with PZN-Cy5 as described for confocal microscopy. The cells were immobilized on Nunc Lab-Tek 8-well chambered coverglass (Sigma-Aldrich) coated with $0.1 \%(\mathrm{w} / \mathrm{V})$ poly-L-lysine (SigmaAldrich). After 10 min incubation, unattached cells were removed by washing chambers with sterile PBS. Chambers were filled with $500 \mu \mathrm{L}$ imaging buffer $(10 \mathrm{mM} \mathrm{NaCl}, 50 \mathrm{mM}$ Tris- $\mathrm{HCl}$ (pH 8.5), 10\% W/v glucose). Immediately prior to imaging, cysteamine (SigmaAldrich, $10 \mathrm{mM}$ final concentration), catalase (EMD Millipore, $909 \mathrm{U} / \mathrm{mL}$ ), and pyranose oxidase (Sigma-Aldrich, $4.44 \mathrm{U} / \mathrm{mL}$ ) were added to the imaging buffer. 3D super-resolution microscopy was performed as described previously. ${ }^{(77,78)}$ Briefly, samples were imaged using an Olympus IX-71 inverted microscope outfitted with a 100× NA 1.4 SaPo oil objective. Mechanical shutters (LS6T2, Unibliz) were used to alternatively excite the sample with a red laser (DL640-100-AL-O, Crystalaser) and reactivate Cy5 with a violet laser (405 $\mathrm{nm}, 20 \mathrm{~mW}$, Spectra Physics Excelsor). The lasers were expanded by $7.5 \times$, reflected by a dichoroic mirror (Semrock FF408/504/581/667/762-Di01-25X36), and sent to the sample chamber with a focusing lens that also creates an incidental angle slightly smaller than the total internal reflection angle, reducing the background signal while allowing illumination of several hundred $\mathrm{nm}$ along the $\mathrm{z}$-axis. The emission signal from the sample was passed through an emission filter (Semrock FF01-594/730-25) and two additional notch filters (Semrock NF01-568/647-25X5.0 and NF01-568U-25), and was imaged on an EMCCD camera (DV887ECS-BV, Andor Tech). A cylindrical lens (SCX-50.8-1000.0-UVSLMF-520-820, CVI Melles Griot, $2 \mathrm{~m}$ focal length) in the emission beam path induced astigmatism for 3D detection. ${ }^{(51)}$ ASI CRISP (Applied Scientific Instrumentation) and a piezo-objective (PI P-721.10) were used to compensate for vertical drift during data 
collection. The horizontal drift was corrected in the post data acquisition step by the analysis software utilizing the correlation function. ${ }^{(79)}$ The data analysis software was provided by Xiaowei Zhuang ${ }^{(77)}$ and modified for 3D imaging.

\section{Selection of spontaneous PZN-resistant mutants}

Spontaneous PZN-resistant mutants were generated by plating $2 \times 10^{8} \mathrm{~B}$. anthracis Sterne 7702 cells grown to stationary phase onto a PZN plate containing $4 \times$ PZN MIC. Surviving colonies were tested for sustained PZN resistance via microbroth dilution as described above. Resistant mutants PR01, PR02, PR05, and PR06 were subjected to genomic DNA isolation as follows: $3 \times 10 \mathrm{~mL}$ cultures of each strain were grown to stationary phase, harvested, and resuspended in $400 \mu \mathrm{L}$ of water. Cells were lysed with $50 \mu \mathrm{L}$ of $10 \%$ SDS and $5 \mu \mathrm{L}$ of $20 \mathrm{mg} / \mathrm{mL}$ RNase solution at $22{ }^{\circ} \mathrm{C}$ for $5 \mathrm{~min}$. DNA was isolated via 25:24:1 phenol/chloroform/isoamyl alcohol extraction, followed by addition of 24:1 chloroform/ isoamyl alcohol. DNA precipitation via cold isopropyl alcohol and a subsequent $70 \%$ ethyl alcohol wash resulted in purified gDNA.

After genetic deletion of bas4114-4117(described in the supporting methods), a second round of spontaneously resistant mutants to PZN were selected and isolated as above. Serialpassage mutants were isolated as previously described, ${ }^{(59)}$ starting with three independent cultures of an $\mathrm{OD}_{600}$ of 0.1 B. anthracis Sterne $7702 \Delta$ bas4114-4117 in $0.25 \mu \mathrm{g} / \mathrm{mL}(0.25 \times$ MIC) PZN LB. Cultures that grew were diluted to an $\mathrm{OD}_{600}$ of 0.1 and subjected to increased concentrations of PZN, until cultures were resistant to $64 \mu \mathrm{g} / \mathrm{mL}$. Cultures were serially passaged onto PZN-free medium to confirm mutant stability. Genomic DNA was isolated as described above. All mutants derived from the $\Delta$ bas4114-4117 deletion strain were sequenced as described and assembled via CLC Genomics Workbench and SNP analysis was performed with Mauve version 2.3.1.

\section{Whole genome sequencing and assembly}

Genomic libraries for resequencing were prepared using the TruSeq DNAseq Sample prep kit (Illumina, San Diego, CA). Sequencing was performed on a HiSeq 2000 with Version 3 Chemistry for $1 \times 100$ cycles. SNP and DIP discovery was performed with two different methods. Regarding PR02, PR05, and PR06, CLC Genomics Workbench SNP and DIP discovery pipelines were employed using with the publicly available $B$. anthracis str. Sterne genome NC_005945.1 as a reference. PR01 required de novo assembly with IDBA UD version 1.0.9, followed by whole genome alignment and SNP discovery using Mauve version 2.3.1. Resistant mutants PR03 and PR04 were selected separately and underwent Sanger sequencing after PCR amplification of bas4114 and sequencing with the BamHIBAS4114-f primer (Table S5). The WGS data discussed in this publication have been deposited in NCBI's GenBank and are accessible via BioProject accession number PRJNA295544. Within this BioProject are individual accession numbers for each $B$. anthracis strain (taxId:1392) for which whole genome sequencing was performed: CP012720, PR01; CP012721, PR02; CP012722, PR05; CP012723, PR06; CP012724, PR07; CP012725, PR08; CP012726, PR09-1; CP012727, PR09-4; CP012728, PR10-4; CP012730, Parent1 (for PR01 through PR06); CP012729, Parent2 (for PR07 through PR10-4. 


\section{Effect of cardiolipin on fluorescence intensity}

Three independent stationary phase cultures of $B$. anthracis Sterne 7702 were used to inoculate fresh $\mathrm{LB}(200 \mu \mathrm{L}$ into $5 \mathrm{~mL} \mathrm{LB})$ and the new cultures were grown to $\mathrm{OD}_{600} 0.5$ at $37^{\circ} \mathrm{C}$ with shaking. Samples were prepared by diluting $10 \mu \mathrm{L}$ aliquots of culture to $1 \mathrm{~mL}$ in PBS containing $1 \mathrm{nM}$ PZN-Cy5 and vehicle (EtOH), $10 \mu \mathrm{g} / \mathrm{mL}$ CL (Sigma Aldrich), or 100 $\mu \mathrm{g} / \mathrm{mL} \mathrm{CL}$. After mixing at $22{ }^{\circ} \mathrm{C}$ for $30 \mathrm{~min}$, cells were analyzed by flow cytometry as described above for differences in PZN-Cy5 fluorescence intensity. Geometric means were normalized to the control samples.

\section{Cardiolipin quantification from total lipid extracts}

Cultures of B. anthracis Sterne 7702, B. anthracis $\Delta$ bas4114-4117, B. anthracis PR09-4, B. anthracis PR10-4, B. subtilis 168, E. faecium U503, and $S$. aureus USA300 (three independent $10 \mathrm{~mL}$ cultures for each strain) were grown for $20 \mathrm{~h}$ at $37^{\circ} \mathrm{C}$. LB containing an additional $1.5 \mathrm{M} \mathrm{NaCl}$ was inoculated with $200 \mu \mathrm{L}$ aliquots of stationary phase cultures of B. subtilis 168 or $0.5 \mathrm{M} \mathrm{NaCl}$ for B. anthracis and B. cereus (three independent $10 \mathrm{~mL}$ cultures for each strain) and grown for $40 \mathrm{~h}$ at $37{ }^{\circ} \mathrm{C}$. The cells were harvested by centrifugation $\left(4000 \times \mathrm{g}, 10 \mathrm{~min}, 4^{\circ} \mathrm{C}\right)$ and resuspended in $5 \mathrm{~mL} \mathrm{2:1} \mathrm{CHCl}_{3}: \mathrm{MeOH}$ and $1.25 \mathrm{~mL}$ PBS, and then extracted for $1 \mathrm{~h}$ at $22^{\circ} \mathrm{C}$. The supernatant was removed after centrifugation $\left(4000 \times \mathrm{g}, 10 \mathrm{~min}, 4^{\circ} \mathrm{C}\right)$ and layers were washed with $1 \mathrm{~mL} \mathrm{CHCl}_{3}$ and $1 \mathrm{~mL}$ PBS. The organic layer was removed and dried by speed vacuum. The crude lipids were redissolved in $200 \mu \mathrm{L} \mathrm{CHCl}_{3}$ and transferred to microfuge tubes, then dried again. The lipids were then dissolved in $20 \mu \mathrm{L} \mathrm{CHCl}_{3}$, spotted $(2 \mu \mathrm{L})$ onto Merck Silica Gel $60 \mathrm{~F}_{254}$ analytical TLC plates and separated using 80:20:5 $\mathrm{CHCl}_{3}: \mathrm{MeOH}: \mathrm{AcOH}$. Pure CL was used as a standard. The plates were imaged using a Bio-Rad ChemiDoc XRS+. ImageJ was used to subtract background and measure spot density to determine percent CL out of total lipid content.

\section{Supplementary Material}

Refer to Web version on PubMed Central for supplementary material.

\section{Acknowledgments}

We are grateful to the Pasteur Institute, BEI Resources, O. Schneewind (U. Chicago), C. Turnbough (U. Alabama Birmingham), U.S. Department of Agriculture, The Caenorhabditis Genetics Center, The Bacillus Genetic Stock Center, S. Shozhamannan (The Naval Medical Research Center), V. Fischetti (Rockefeller U.), C. Marston (Center for Disease Control and Prevention), D. de Mendoza (U. Nacional de Rosario), J. Wells (UCSF), J. Helmann (Cornell U.), M. So (U. Arizona), P. Hergenrother (UIUC), H. Zhao (UIUC), P. Hanna (U. Michigan), and S. Leppla (National Institute of Allergy and Infectious Diseases) for providing strains and plasmids used in this study, Dr. Yao Shao Qin and Dr. Li Zhengqiu (National U. of Singapore) for providing 2-(2-azidoethyl)-2-(but-3-ynyl)-1,3dioxolane, as well as Teresa Abshire, Stephanie Halasohoris, and Lynda Miller (U.S. Army Medical Research Institute for Infectious Diseases, USAMRIID) for providing us with $\gamma$ phage and their assistance. We also thank C. Deane for critical review of this manuscript.

\section{Funding Statement}

This work was supported by the US National Institutes of Health (1R01 GM097142 to DAM) and the Defense Threat Reduction Agency under USAMRIID (project \#922141). KJM, PMB, and JRD were supported in part by a James R. Beck Graduate Research Fellowship, an ACS Medicinal Chemistry Predoctoral Fellowship, and the Carle R. Woese Institute for Genomic Biology Fellows program, respectively. The funders had no role in study design, 
data collection and analysis, decision to publish, or preparation of the manuscript. Opinions, interpretations, conclusions and recommendations are those of the authors and are not necessarily endorsed by the U.S. Army.

\section{Abbreviations}

$\begin{array}{ll}\text { PZN } & \text { plantazolicin } \\ \text { RiPP } & \begin{array}{l}\text { ribosomally synthesized and post-translationally modified peptide natural } \\ \text { product }\end{array} \\ \text { TOMM } & \begin{array}{l}\text { thiazole/oxazole-modified microcin } \\ \text { MOA }\end{array} \\ \text { mode of action } \\ \text { SCWP } & \text { secondary cell wall polysaccharide } \\ \text { CL } & \text { cardiolipin } \\ \text { MIC } & \text { minimum inhibitory concentration } \\ \text { Des } & \text { lipid desaturase } \\ \text { NAO } & 10-\text { N-nonyl acridine orange } \\ \text { RIF } & \text { regions of increased fluidity } \\ \text { DilC12(3) } & 1,1^{\prime} \text {-Didodecyl-3,3, } 3^{\prime}, 3^{\prime} \text {-tetramethylindocarbocyanine perchlorate }\end{array}$

\section{References}

1. de Man P, Verhoeven BA, Verbrugh HA, Vos MC, van den Anker JN. An antibiotic policy to prevent emergence of resistant bacilli. Lancet. 2000; 355:973-978. [PubMed: 10768436]

2. Wilson DN, Harms JM, Nierhaus KH, Schlunzen F, Fucini P. Species-specific antibiotic-ribosome interactions: implications for drug development. Biol Chem. 2005; 386:1239-1252.10.1515/BC. 2005.141 [PubMed: 16336118]

3. Payne DJ. Desperately seeking new antibiotics. Science. 2008; 321:1644-1645.10.1126/science. 1164586 [PubMed: 18801989]

4. Scholz R, Molohon KJ, Nachtigall J, Vater J, Markley AL, Sussmuth RD, Mitchell DA, Borriss R. Plantazolicin, a novel microcin B17/streptolysin S-like natural product from Bacillus amyloliquefaciens FZB42. J Bacteriol. 2011; 193:215-224.10.1128/JB.00784-10 [PubMed: 20971906]

5. Arnison PG, Bibb MJ, Bierbaum G, Bowers AA, Bugni TS, Bulaj G, Camarero JA, Campopiano DJ, Challis GL, Clardy J, Cotter PD, Craik DJ, Dawson M, Dittmann E, Donadio S, Dorrestein PC, Entian KD, Fischbach MA, Garavelli JS, Goransson U, Gruber CW, Haft DH, Hemscheidt TK, Hertweck C, Hill C, Horswill AR, Jaspars M, Kelly WL, Klinman JP, Kuipers OP, Link AJ, Liu W, Marahiel MA, Mitchell DA, Moll GN, Moore BS, Muller R, Nair SK, Nes IF, Norris GE, Olivera BM, Onaka H, Patchett ML, Piel J, Reaney MJ, Rebuffat S, Ross RP, Sahl HG, Schmidt EW, Selsted ME, Severinov K, Shen B, Sivonen K, Smith L, Stein T, Sussmuth RD, Tagg JR, Tang GL, Truman AW, Vederas JC, Walsh CT, Walton JD, Wenzel SC, Willey JM, van der Donk WA. Ribosomally synthesized and post-translationally modified peptide natural products: overview and recommendations for a universal nomenclature. Nat Prod Rep. 2013; 30:108-160.10.1039/ c2np20085f [PubMed: 23165928]

6. Cox CL, Doroghazi JR, Mitchell DA. The genomic landscape of ribosomal peptides containing thiazole and oxazole heterocycles. BMC Genomics. 2015; 16:778.10.1186/s12864-015-2008-0 [PubMed: 26462797]

7. Dunlap CA, Kim SJ, Kwon SW, Rooney AP. Phylogenomic analysis shows that Bacillus amyloliquefaciens subsp. plantarum is a later heterotypic synonym of Bacillus methylotrophicus. Int J Syst Evol Microbiol. 2015; 65:2104-2109.10.1099/ijs.0.000226 [PubMed: 25835027] 
8. Molohon KJ, Melby JO, Lee J, Evans BS, Dunbar KL, Bumpus SB, Kelleher NL, Mitchell DA. Structure determination and interception of biosynthetic intermediates for the plantazolicin class of highly discriminating antibiotics. ACS Chem Biol. 2011; 6:1307-1313.10.1021/cb200339d [PubMed: 21950656]

9. Banala S, Ensle P, Sussmuth RD. Total synthesis of the ribosomally synthesized linear azolecontaining peptide plantazolicin A from Bacillus amyloliquefaciens. Angew Chem Int Ed Engl. 2013; 52:9518-9523.10.1002/anie.201302266 [PubMed: 23761292]

10. Wilson ZE, Fenner S, Ley SV. Total syntheses of linear polythiazole/oxazole plantazolicin A and its biosynthetic precursor plantazolicin B. Angew Chem Int Ed Engl. 2015; 54:12841288.10.1002/anie.201410063 [PubMed: 25424526]

11. Wada H, Williams HE, Moody CJ. Total Synthesis of the Posttranslationally Modified Polyazole Peptide Antibiotic Plantazolicin A. Angew Chem Int Ed Engl. 201510.1002/anie.201507062

12. Deane CD, Melby JO, Molohon KJ, Susarrey AR, Mitchell DA. Engineering unnatural variants of plantazolicin through codon reprogramming. ACS Chem Biol. 2013; 8:1998-2008.10.1021/ cb4003392 [PubMed: 23823732]

13. Lee J, Hao Y, Blair PM, Melby JO, Agarwal V, Burkhart BJ, Nair SK, Mitchell DA. Structural and functional insight into an unexpectedly selective N-methyltransferase involved in plantazolicin biosynthesis. Proc Natl Acad Sci U S A. 2013; 110:12954-12959.10.1073/pnas.1306101110 [PubMed: 23878226]

14. Sharma A, Blair PM, Mitchell DA. Synthesis of plantazolicin analogues enables dissection of ligand binding interactions of a highly selective methyltransferase. Org Lett. 2013; 15:50765079.10.1021/ol402444a [PubMed: 24063702]

15. Hao Y, Blair PM, Sharma A, Mitchell DA, Nair SK. Insights into methyltransferase specificity and bioactivity of derivatives of the antibiotic plantazolicin. ACS Chem Biol. 2015; 10:12091216.10.1021/cb501042a [PubMed: 25635336]

16. Lee SW, Mitchell DA, Markley AL, Hensler ME, Gonzalez D, Wohlrab A, Dorrestein PC, Nizet V, Dixon JE. Discovery of a widely distributed toxin biosynthetic gene cluster. Proc Natl Acad Sci U S A. 2008; 105:5879-5884.10.1073/pnas.0801338105 [PubMed: 18375757]

17. Rasko DA, Altherr MR, Han CS, Ravel J. Genomics of the Bacillus cereus group of organisms. FEMS Microbiol Rev. 2005; 29:303-329.10.1016/j.femsre.2004.12.005 [PubMed: 15808746]

18. Kolsto AB, Tourasse NJ, Okstad OA. What sets Bacillus anthracis apart from other Bacillus species? Annu Rev Microbiol. 2009; 63:451-476.10.1146/annurev.micro.091208.073255 [PubMed: 19514852]

19. Mesnage S, Fontaine T, Mignot T, Delepierre M, Mock M, Fouet A. Bacterial SLH domain proteins are non-covalently anchored to the cell surface via a conserved mechanism involving wall polysaccharide pyruvylation. EMBO J. 2000; 19:4473-4484.10.1093/emboj/19.17.4473 [PubMed: 10970841]

20. Kern J, Ryan C, Faull K, Schneewind O. Bacillus anthracis surface-layer proteins assemble by binding to the secondary cell wall polysaccharide in a manner that requires $\operatorname{csaB}$ and $\operatorname{tag} O$. J Mol Biol. 2010; 401:757-775.10.1016/j.jmb.2010.06.059 [PubMed: 20603129]

21. Weidenmaier C, Peschel A. Teichoic acids and related cell-wall glycopolymers in Gram-positive physiology and host interactions. Nat Rev Microbiol. 2008; 6:276-287.10.1038/nrmicro1861 [PubMed: 18327271]

22. Ganguly J, Low LY, Kamal N, Saile E, Forsberg LS, Gutierrez-Sanchez G, Hoffmaster AR, Liddington R, Quinn CP, Carlson RW, Kannenberg EL. The secondary cell wall polysaccharide of Bacillus anthracis provides the specific binding ligand for the C-terminal cell wall-binding domain of two phage endolysins, PlyL and PlyG. Glycobiology. 2013; 23:820-832.10.1093/glycob/cwt019 [PubMed: 23493680]

23. Schuch R, Pelzek AJ, Raz A, Euler CW, Ryan PA, Winer BY, Farnsworth A, Bhaskaran SS, Stebbins CE, Xu Y, Clifford A, Bearss DJ, Vankayalapati H, Goldberg AR, Fischetti VA. Use of a bacteriophage lysin to identify a novel target for antimicrobial development. PLoS One. 2013; 8:e60754.10.1371/journal.pone.0060754 [PubMed: 23593301] 
24. Ezzell JW, Abshire TG, Little SF, Lidgerding BC, Brown C. Identification of Bacillus anthracis by using monoclonal antibody to cell wall galactose- $N$-acetylglucosamine polysaccharide. J Clin Microbiol. 1990; 28:223-231. [PubMed: 2107201]

25. Abshire TG, Brown JE, Ezzell JW. Production and validation of the use of gamma phage for identification of Bacillus anthracis. J Clin Microbiol. 2005; 43:4780-4788.10.1128/JCM. 43.9.4780-4788.2005 [PubMed: 16145141]

26. Kan S, Fornelos N, Schuch R, Fischetti VA. Identification of a ligand on the Wip1 bacteriophage highly specific for a receptor on B. anthracis. J Bacteriol. 201310.1128/JB.00655-13

27. Hoffmaster AR, Hill KK, Gee JE, Marston CK, De BK, Popovic T, Sue D, Wilkins PP, Avashia SB, Drumgoole R, Helma CH, Ticknor LO, Okinaka RT, Jackson PJ. Characterization of Bacillus cereus isolates associated with fatal pneumonias: strains are closely related to Bacillus anthracis and harbor $B$ anthracis virulence genes. J Clin Microbiol. 2006; 44:3352-3360.10.1128/JCM. 00561-06 [PubMed: 16954272]

28. Schuch R, Fischetti VA. Detailed genomic analysis of the Wbeta and gamma phages infecting Bacillus anthracis: implications for evolution of environmental fitness and antibiotic resistance. $\mathrm{J}$ Bacteriol. 2006; 188:3037-3051.10.1128/JB.188.8.3037-3051.2006 [PubMed: 16585764]

29. Marston CK, Gee JE, Popovic T, Hoffmaster AR. Molecular approaches to identify and differentiate Bacillus anthracis from phenotypically similar Bacillus species isolates. BMC Microbiol. 2006; 6:22.10.1186/1471-2180-6-22 [PubMed: 16515693]

30. Davison S, Couture-Tosi E, Candela T, Mock M, Fouet A. Identification of the Bacillus anthracis (gamma) phage receptor. J Bacteriol. 2005; 187:6742-6749.10.1128/JB.187.19.6742-6749.2005 [PubMed: 16166537]

31. Hoffmaster AR, Ravel J, Rasko DA, Chapman GD, Chute MD, Marston CK, De BK, Sacchi CT, Fitzgerald C, Mayer LW, Maiden MC, Priest FG, Barker M, Jiang L, Cer RZ, Rilstone J, Peterson SN, Weyant RS, Galloway DR, Read TD, Popovic T, Fraser CM. Identification of anthrax toxin genes in a Bacillus cereus associated with an illness resembling inhalation anthrax. Proc Natl Acad Sci U S A. 2004; 101:8449-8454.10.1073/pnas.0402414101 [PubMed: 15155910]

32. Burkett-Cadena M, Kokalis-Burelle N, Lawrence KS, van Santen E, Kloepper JW. Suppressiveness of root-knot nematodes mediated by rhizobacteria. Biological Control. 2008; 47:55-59. doi: 10:1016.

33. Liu Z, Budiharjo A, Wang P, Shi H, Fang J, Borriss R, Zhang K, Huang X. The highly modified microcin peptide plantazolicin is associated with nematicidal activity of Bacillus amyloliquefaciens FZB42. Appl Microbiol Biotechnol. 2013; 97:10081-10090.10.1007/ s00253-013-5247-5 [PubMed: 24085393]

34. Ziegler S, Pries V, Hedberg C, Waldmann H. Target identification for small bioactive molecules: finding the needle in the haystack. Angew Chem Int Ed Engl. 2013; 52:2744-2792.10.1002/anie. 201208749 [PubMed: 23418026]

35. Burdine L, Kodadek T. Target identification in chemical genetics: the (often) missing link. Chem Biol. 2004; 11:593-597.10.1016/j.chembiol.2004.05.001 [PubMed: 15157870]

36. Hobbs JK, Miller K, O’Neill AJ, Chopra I. Consequences of daptomycin-mediated membrane damage in Staphylococcus aureus. J Antimicrob Chemother. 2008; 62:1003-1008.10.1093/jac/ dkn321 [PubMed: 18669516]

37. Sahl HG, Brandis H. Mode of action of the staphylococcin-like peptide Pep 5 and culture conditions effecting its activity. Zentralbl Bakteriol Mikrobiol Hyg A. 1982; 252:166-175. [PubMed: 6181629]

38. Brazas MD, Hancock RE. Using microarray gene signatures to elucidate mechanisms of antibiotic action and resistance. Drug Discov Today. 2005; 10:1245-1252.10.1016/S1359-6446(05)03566-X [PubMed: 16213417]

39. Shaw KJ, Morrow BJ. Transcriptional profiling and drug discovery. Curr Opin Pharmacol. 2003; 3:508-512.10.1016/S1471-4892(03)00110-3 [PubMed: 14559096]

40. Li XZ, Nikaido H. Efflux-mediated drug resistance in bacteria: an update. Drugs. 2009; 69:15551623.10.2165/11317030-000000000-00000 [PubMed: 19678712]

41. Jordan S, Hutchings MI, Mascher T. Cell envelope stress response in Gram-positive bacteria. FEMS Microbiol Rev. 2008; 32:107-146.10.1111/j.1574-6976.2007.00091.x [PubMed: 18173394] 
42. Wecke T, Zuhlke D, Mader U, Jordan S, Voigt B, Pelzer S, Labischinski H, Homuth G, Hecker M, Mascher T. Daptomycin versus Friulimicin B: in-depth profiling of Bacillus subtilis cell envelope stress responses. Antimicrob Agents Chemother. 2009; 53:1619-1623.10.1128/AAC.01046-08 [PubMed: 19164157]

43. Martin M, de Mendoza D. Regulation of Bacillus subtilis DesK thermosensor by lipids. Biochem J. 2013; 451:269-275.10.1042/BJ20121825 [PubMed: 23356219]

44. Novo D, Perlmutter NG, Hunt RH, Shapiro HM. Accurate flow cytometric membrane potential measurement in bacteria using diethyloxacarbocyanine and a ratiometric technique. Cytometry. 1999; 35:55-63. [PubMed: 10554181]

45. Ruhr E, Sahl HG. Mode of action of the peptide antibiotic nisin and influence on the membrane potential of whole cells and on cytoplasmic and artificial membrane vesicles. Antimicrob Agents Chemother. 1985; 27:841-845. [PubMed: 4015074]

46. Silverman JA, Perlmutter NG, Shapiro HM. Correlation of daptomycin bactericidal activity and membrane depolarization in Staphylococcus aureus. Antimicrob Agents Chemother. 2003; 47:2538-2544.10.1128/AAC.47.8.2538-2544.2003 [PubMed: 12878516]

47. Epand RM, Walker C, Epand RF, Magarvey NA. Molecular mechanisms of membrane targeting antibiotics. Biochim Biophys Acta. 201510.1016/j.bbamem.2015.10.018

48. Berenbaum MC. What is synergy? Pharmacol Rev. 1989; 41:93-141. [PubMed: 2692037]

49. Tiyanont K, Doan T, Lazarus MB, Fang X, Rudner DZ, Walker S. Imaging peptidoglycan biosynthesis in Bacillus subtilis with fluorescent antibiotics. Proc Natl Acad Sci U S A. 2006; 103:11033-11038.10.1073/pnas.0600829103 [PubMed: 16832063]

50. Bindman NA, van der Donk WA. A general method for fluorescent labeling of the N-termini of lanthipeptides and its application to visualize their cellular localization. J Am Chem Soc. 2013; 135:10362-10371.10.1021/ja4010706 [PubMed: 23789944]

51. Huang B, Wang W, Bates M, Zhuang X. Three-dimensional super-resolution imaging by stochastic optical reconstruction microscopy. Science. 2008; 319:810-813.10.1126/science.1153529 [PubMed: 18174397]

52. Nguyen-Mau SM, Oh SY, Kern VJ, Missiakas DM, Schneewind O. Secretion genes as determinants of Bacillus anthracis chain length. J Bacteriol. 2012; 194:3841-3850.10.1128/JB. 00384-12 [PubMed: 22609926]

53. Silver LL. Challenges of antibacterial discovery. Clin Microbiol Rev. 2011; 24:71-109.10.1128/ CMR.00030-10 [PubMed: 21233508]

54. Deng W, Li C, Xie J. The underling mechanism of bacterial TetR/AcrR family transcriptional repressors. Cell Signal. 2013; 25:1608-1613.10.1016/j.cellsig.2013.04.003 [PubMed: 23602932]

55. Poelarends GJ, Mazurkiewicz P, Konings WN. Multidrug transporters and antibiotic resistance in Lactococcus lactis. Biochim Biophys Acta. 2002; 1555:1-7. [PubMed: 12206883]

56. Collins B, Curtis N, Cotter PD, Hill C, Ross RP. The ABC transporter AnrAB contributes to the innate resistance of Listeria monocytogenes to nisin, bacitracin, and various beta-lactam antibiotics. Antimicrob Agents Chemother. 2010; 54:4416-4423.10.1128/AAC.00503-10 [PubMed: 20643901]

57. Yang DC, Peters NT, Parzych KR, Uehara T, Markovski M, Bernhardt TG. An ATP-binding cassette transporter-like complex governs cell-wall hydrolysis at the bacterial cytokinetic ring. P Natl Acad Sci USA. 2011; 108:E1052-E1060.10.1073/pnas.1107780108

58. Garti-Levi S, Hazan R, Kain J, Fujita M, Ben-Yehuda S. The FtsEX ABC transporter directs cellular differentiation in Bacillus subtilis. Mol Microbiol. 2008; 69:1018-1028.10.1111/j. 1365-2958.2008.06340.x [PubMed: 18573177]

59. Friedman L, Alder JD, Silverman JA. Genetic changes that correlate with reduced susceptibility to daptomycin in Staphylococcus aureus. Antimicrob Agents Chemother. 2006; 50:21372145.10.1128/AAC.00039-06 [PubMed: 16723576]

60. Lee JY, Janes BK, Passalacqua KD, Pfleger BF, Bergman NH, Liu H, Hakansson K, Somu RV, Aldrich CC, Cendrowski S, Hanna PC, Sherman DH. Biosynthetic analysis of the petrobactin siderophore pathway from Bacillus anthracis. J Bacteriol. 2007; 189:1698-1710.10.1128/JB. 01526-06 [PubMed: 17189355] 
61. Zhang T, Muraih JK, Tishbi N, Herskowitz J, Victor RL, Silverman J, Uwumarenogie S, Taylor SD, Palmer M, Mintzer E. Cardiolipin prevents membrane translocation and permeabilization by daptomycin. J Biol Chem. 2014; 289:11584-11591.10.1074/jbc.M114.554444 [PubMed: 24616102]

62. Palmer KL, Daniel A, Hardy C, Silverman J, Gilmore MS. Genetic basis for daptomycin resistance in Enterococci. Antimicrob Agents Chemother. 2011; 55:3345-3356.10.1128/AAC.00207-11 [PubMed: 21502617]

63. Mileykovskaya E, Dowhan W. Visualization of phospholipid domains in Escherichia coli by using the cardiolipin-specific fluorescent dye 10-N-nonyl acridine orange. J Bacteriol. 2000; 182:11721175.10.1128/JB.182.4.1172-1175.2000 [PubMed: 10648548]

64. Mileykovskaya E, Dowhan W. Cardiolipin membrane domains in prokaryotes and eukaryotes. Biochim Biophys Acta. 2009; 1788:2084-2091.10.1016/j.bbamem.2009.04.003 [PubMed: 19371718]

65. Strahl H, Burmann F, Hamoen LW. The actin homologue MreB organizes the bacterial cell membrane. Nat Commun. 2014; 5:3442.10.1038/ncomms4442 [PubMed: 24603761]

66. Romantsov T, Guan Z, Wood JM. Cardiolipin and the osmotic stress responses of bacteria. Biochim Biophys Acta. 2009; 1788:2092-2100.10.1016/j.bbamem.2009.06.010 [PubMed: 19539601]

67. Peters AC, Thomas L, Wimpenny JW. Effects of salt concentration on bacterial growth on plates with gradients of pH and temperature. FEMS Microbiol Lett. 1991; 61:309-314. [PubMed: 1903754]

68. Unsay JD, Cosentino K, Subburaj Y, Garcia-Saez AJ. Cardiolipin effects on membrane structure and dynamics. Langmuir. 2013; 29:15878-15887.10.1021/la402669z [PubMed: 23962277]

69. Aguilar PS, Cronan JE Jr, de Mendoza D. A Bacillus subtilis gene induced by cold shock encodes a membrane phospholipid desaturase. J Bacteriol. 1998; 180:2194-2200. [PubMed: 9555904]

70. Hotta K, Kim CY, Fox DT, Koppisch AT. Siderophore-mediated iron acquisition in Bacillus anthracis and related strains. Microbiology. 2010; 156:1918-1925.10.1099/mic.0.039404-0 [PubMed: 20466767]

71. Steil L, Hoffmann T, Budde I, Volker U, Bremer E. Genome-wide transcriptional profiling analysis of adaptation of Bacillus subtilis to high salinity. J Bacteriol. 2003; 185:6358-6370. [PubMed: 14563871]

72. Hoffmann T, Schutz A, Brosius M, Volker A, Volker U, Bremer E. High-salinity-induced iron limitation in Bacillus subtilis. J Bacteriol. 2002; 184:718-727. [PubMed: 11790741]

73. Ohta A, Obara T, Asami Y, Shibuya I. Molecular cloning of the cls gene responsible for cardiolipin synthesis in Escherichia coli and phenotypic consequences of its amplification. J Bacteriol. 1985; 163:506-514. [PubMed: 2991193]

74. Kellogg DS Jr, Peacock WL Jr, Deacon WE, Brown L, Pirkle DI. Neisseria gonorrhoeae. I. Virulence genetically linked to clonal variation. J Bacteriol. 1963; 85:1274-1279. [PubMed: 14047217]

75. Clinical and Laboratory Standards Institute. Methods for Dilution Antimicrobial Susceptibility Tests for Bacteria that Grow Aerobically-Seventh Edition: Approved Standard. CLSI; Wayne, PA, USA: 2006.

76. McClure R, Balasubramanian D, Sun Y, Bobrovskyy M, Sumby P, Genco CA, Vanderpool CK, Tjaden B. Computational analysis of bacterial RNA-Seq data. Nucleic Acids Res. 2013; 41:e140.10.1093/nar/gkt444 [PubMed: 23716638]

77. Rust MJ, Bates M, Zhuang X. Sub-diffraction-limit imaging by stochastic optical reconstruction microscopy (STORM). Nat Methods. 2006; 3:793-795.10.1038/nmeth929 [PubMed: 16896339]

78. Fei J, Singh D, Zhang Q, Park S, Balasubramanian D, Golding I, Vanderpool CK, Ha T. RNA biochemistry. Determination of in vivo target search kinetics of regulatory noncoding RNA. Science. 2015; 347:1371-1374.10.1126/science.1258849 [PubMed: 25792329]

79. Bates M, Huang B, Dempsey GT, Zhuang X. Multicolor super-resolution imaging with photoswitchable fluorescent probes. Science. 2007; 317:1749-1753.10.1126/science.1146598 [PubMed: 17702910] 


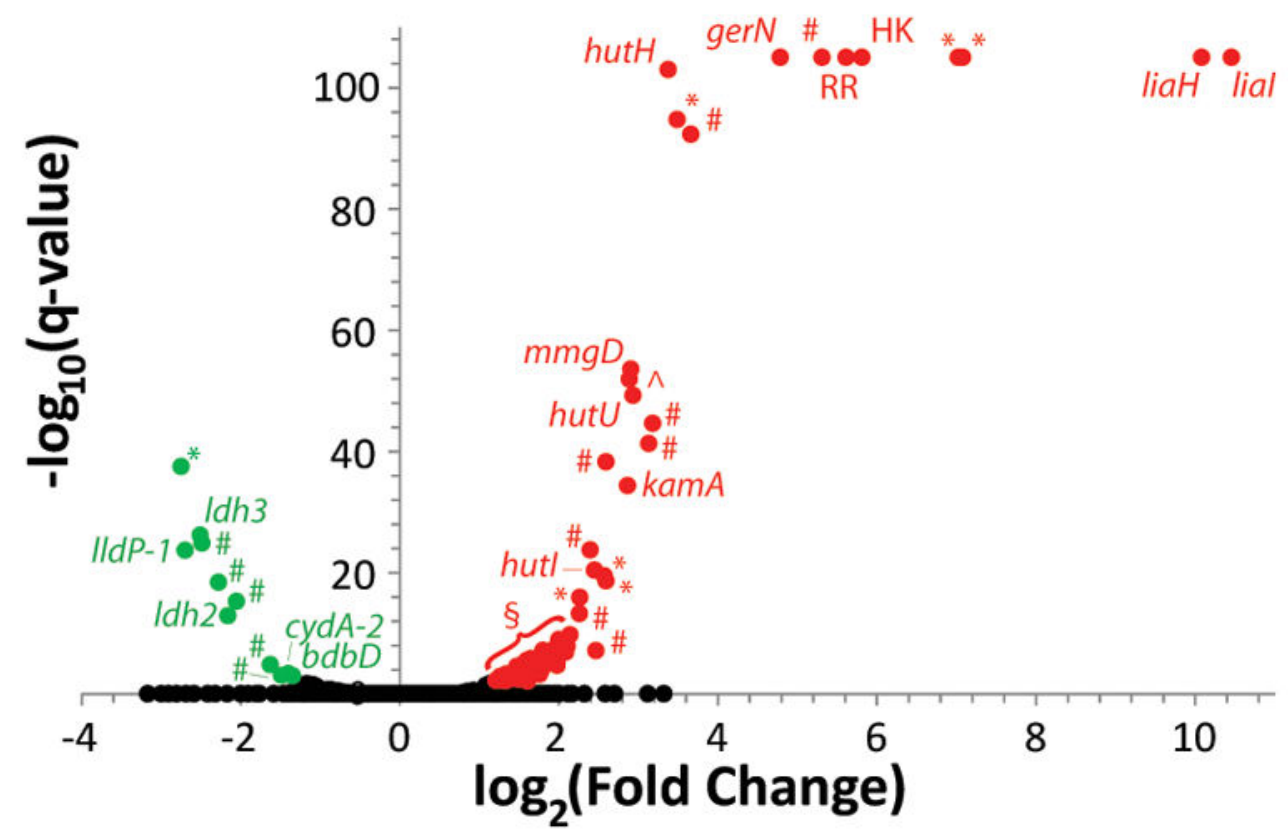

Figure 1. 
a

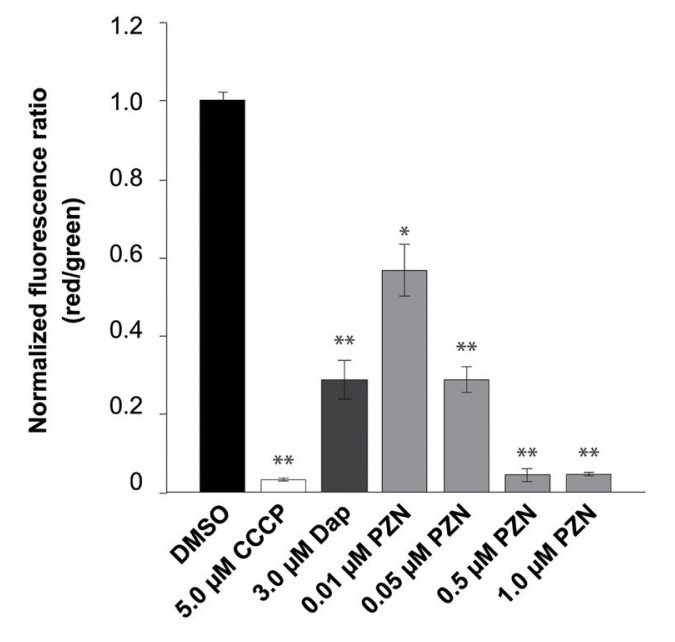

b
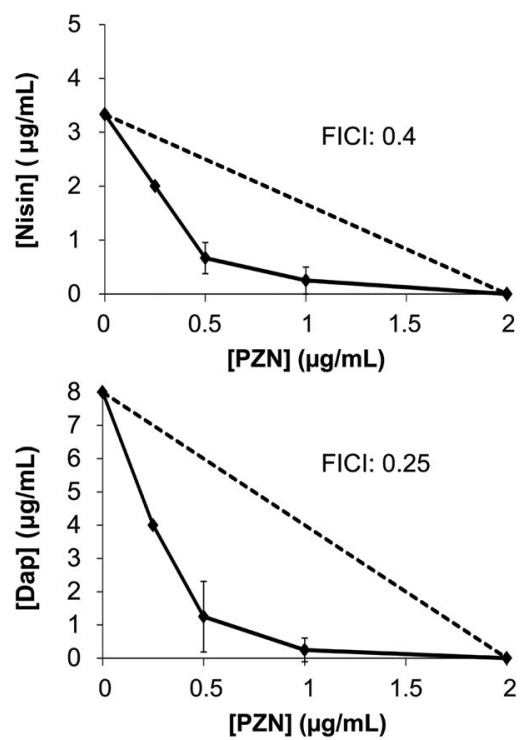

Figure 2. 

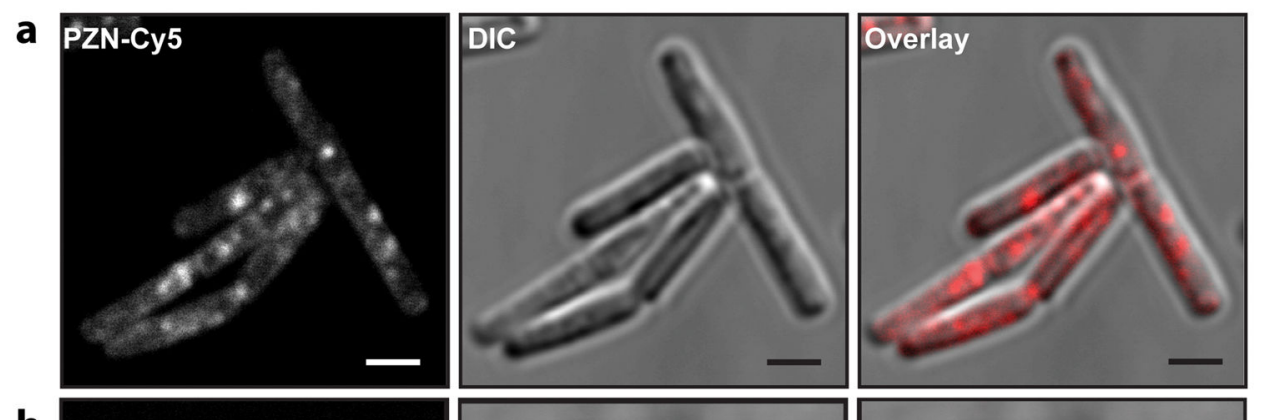

b
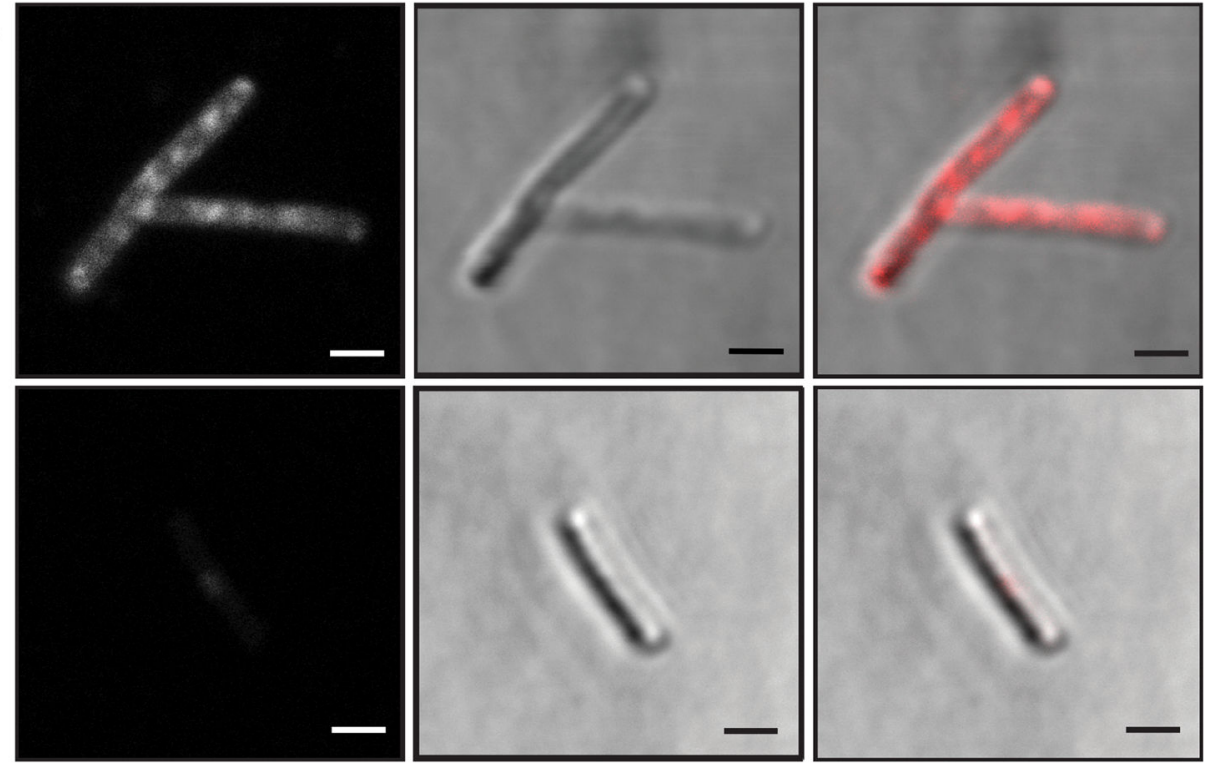

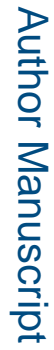

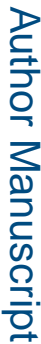

Figure 3.

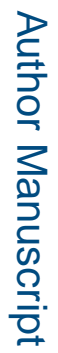




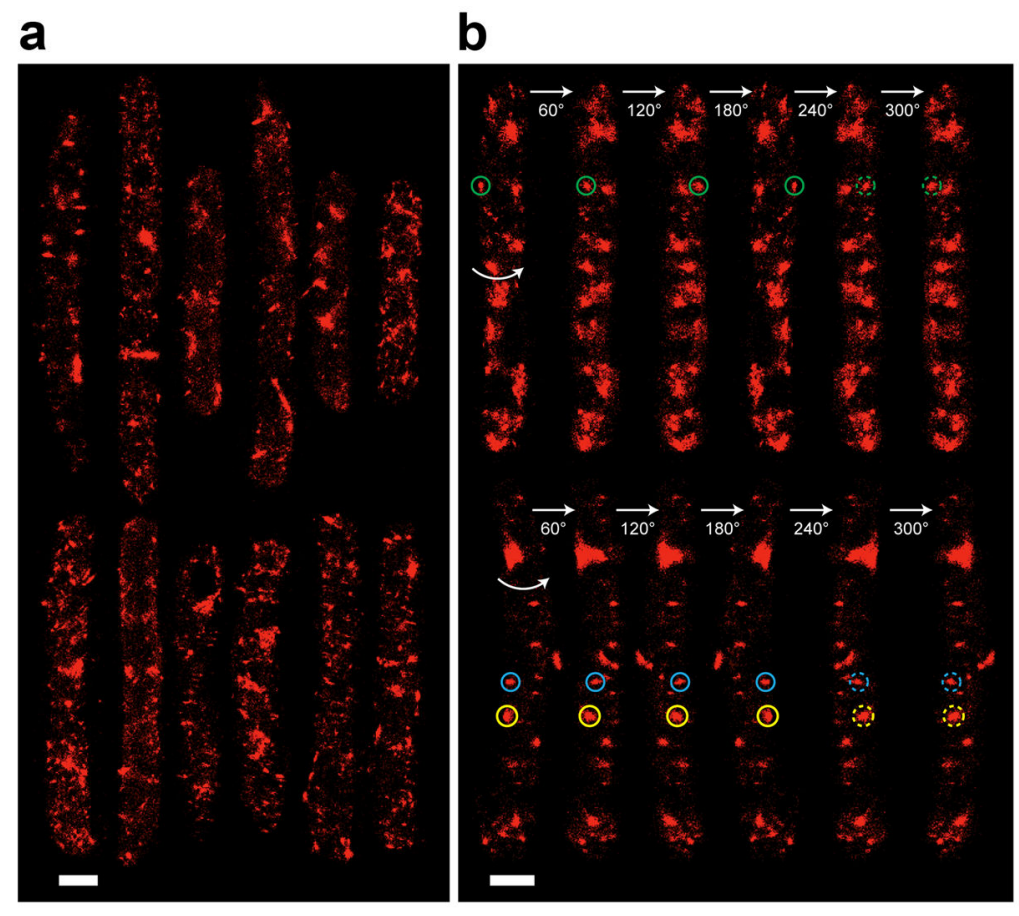

Figure 4. 
a

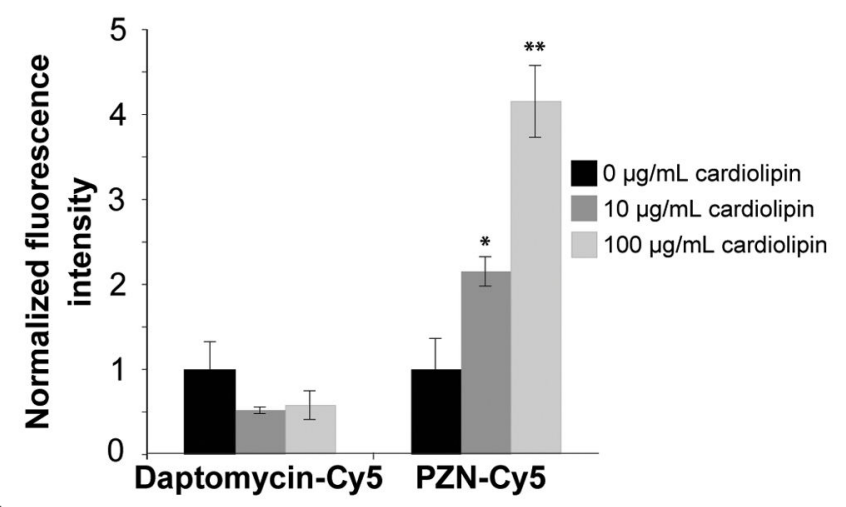

b

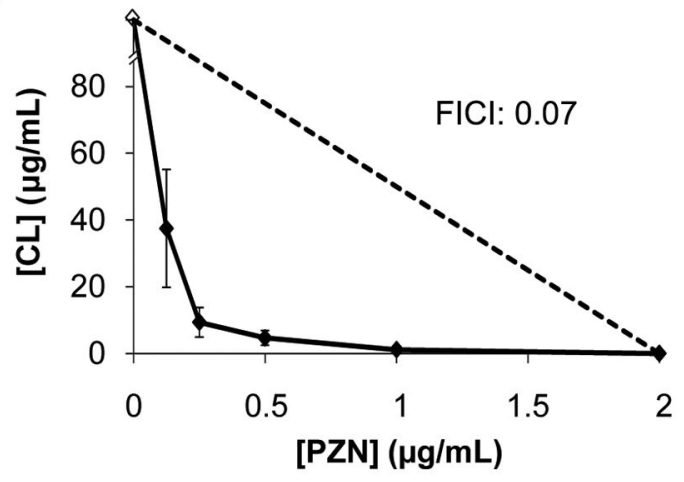

Figure 5. 


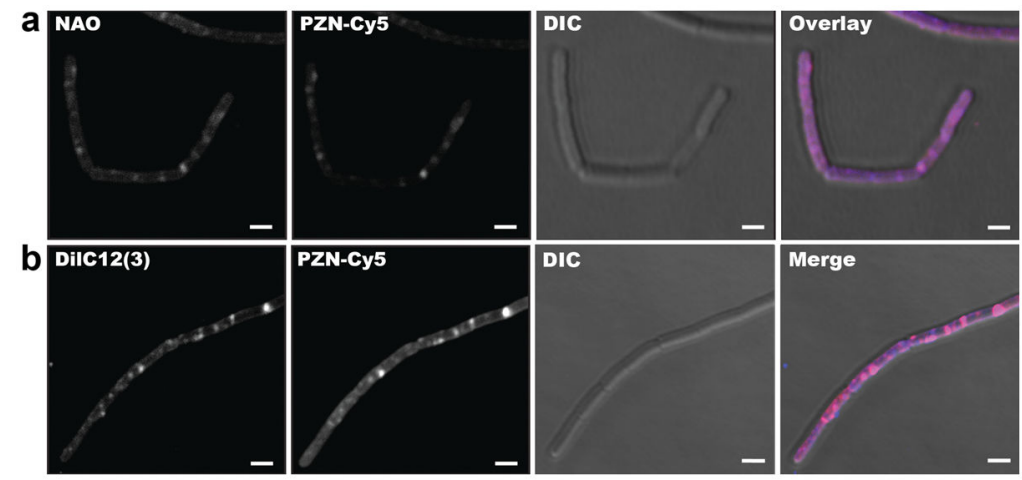

Figure 6. 


\section{Table 1}

Susceptibility of Bacillus sp. to $\gamma$ phage and PZN

\begin{tabular}{|c|c|c|c|}
\hline$\underline{\text { Strain }}$ & $\gamma$ Phage $^{a}$ & $\underline{\mathbf{P Z N}}^{b}$ & $\underline{\text { Source }}^{c}$ \\
\hline B. anthracis Sterne 7702 & +++ & 1 & USDA \\
\hline B. anthracis Sterne $34 \mathrm{~F} 2$ A0517-1 ${ }^{d}$ & +++ & 2 & BEI \\
\hline B. cereus $2002013145^{e}$ & +++ & $>64$ & $\mathrm{CDC}$ \\
\hline B. cereus $2002013146^{e}$ & +++ & $>64$ & $\mathrm{CDC}$ \\
\hline B. cereus $2002013100^{e}$ & ++ & $>64$ & $\mathrm{CDC}$ \\
\hline B. cereus $2000031002^{e}$ & +++ & $>64$ & $\mathrm{CDC}$ \\
\hline B. cereus ATCC 4342 & + & $>64$ & ATCC \\
\hline B. cereus ATCC 7064 & + & $>64$ & ATCC \\
\hline B. cereus CDC 32805 & + & $>64$ & (26) \\
\hline B. cereus $\mathrm{G} 9241$ & - & 8 & BEI \\
\hline B. megaterium 899 & - & 32 & BGSC \\
\hline B. mycoides $96 / 3308$ & - & $>64$ & BGSC \\
\hline
\end{tabular}

${ }^{a}$ Plus signs indicate the level of phage sensitivity, with +++ representing the most sensitive and - indicating complete resistance to the phage

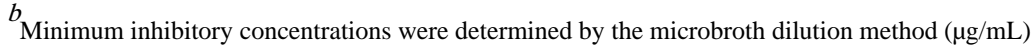

${ }^{c}$ Abbreviations: USDA, United States Department of Agriculture; BEI, Biodefense and Emerging Infections Research Resources Repository; CDC, United States Centers for Disease Control and Prevention; ATCC, American Type Culture Collection; BGSC, Bacillus Genetic Stock Center

dLLNL A0517 was obtained from BEI as a mixture of two colony types. A0517_1 was confirmed to be devoid of pXO1 by PCR (Figure S2)

$e_{\text {Strains identified by multilocus sequence typing analysis }}(27)$ 
Table 2

PZN-resistant mutants of B. anthracis Sterne $\Delta$ bas4114-4117

\begin{tabular}{|c|c|c|c|c|}
\hline$\underline{\text { Strain }}$ & Mutation & Annotation & Consequence & $\underline{\operatorname{MIC}}^{a}$ \\
\hline PR07 & bas5034: A425G & cell division $\mathrm{ABC}$ transporter, FtsE & E142G & 8 \\
\hline PR08 & bas5034: G270T & cell division $\mathrm{ABC}$ transporter, FtsE & L90F & 32 \\
\hline PR09-1 & bas1659: G190C & CitB RR $b_{/ \text {luxR family }}$ & V64L & 16 \\
\hline \multirow[t]{2}{*}{ PR09-4 } & bas1659: G190C & CitB RR $b_{/ l u x R}$ family & V64L & $>64$ \\
\hline & bas1662: A638G & $\mathrm{ABC}$ transporter permease & $\mathrm{H} 213 \mathrm{R}$ & \\
\hline \multirow[t]{3}{*}{ PR10-4 } & bas1659: $\mathrm{C} 248 \mathrm{~T}$ & CitB RR $b_{/ l u x R}$ family & $\mathrm{T} 83 \mathrm{M}$ & 64 \\
\hline & bas1663: $\mathrm{C} 1127 \mathrm{~T}$ & $\mathrm{ABC}$ transporter permease & $\mathrm{A} 376 \mathrm{~V}$ & \\
\hline & bas1842: A43G & petrobactin biosynthesis, AsbE & $\mathrm{S} 15 \mathrm{G}$ & \\
\hline
\end{tabular}

${ }^{a}$ MICs were determined by microbroth dilution assay, measured in $\mu \mathrm{g} / \mathrm{mL}$

$b_{\text {Response regulator }}$ 
Table 3

Effect of $B$. anthracis Sterne mutations and growth conditions on PZN susceptibility and cardiolipin content of bacterial membranes

\begin{tabular}{|c|c|c|c|}
\hline$\underline{\text { Strain }}$ & $\underline{\mathrm{MIC}}^{a}$ & {$[\mathrm{NaCl}]^{b}$} & $\underline{\% \mathrm{CL}^{c}}$ \\
\hline B. anthracis Sterne 7702 & 1 & 0.17 & $8.8 \pm 4.0$ \\
\hline B. anthracis Sterne 7702 & 0.25 & 0.67 & $12.2 \pm 3.4$ \\
\hline B. anthracis Sterne $7702 \Delta$ bas $4114-4117$ & 1 & 0.17 & $11.5 \pm 1.3$ \\
\hline B. anthracis PR09-4 & $>64$ & 0.17 & $14.3 \pm 1.7$ \\
\hline B. anthracis PR09-4 & 32 & 0.67 & $16.3 \pm 0.9$ \\
\hline B. anthracis PR10-4 & 64 & 0.17 & $10.7 \pm 2.3$ \\
\hline B. anthracis PR10-4 & 16 & 0.67 & $11.0 \pm 4.3$ \\
\hline B. subtilis 168 & $>128$ & 0.17 & $16.7 \pm 0.7$ \\
\hline B. subtilis 168 & 32 & 1.67 & $24.1 \pm 0.9^{*}$ \\
\hline B. cereus $\mathrm{E} 33 \mathrm{~L}$ & $>64$ & 0.17 & $11.2 \pm 2.0$ \\
\hline B. cereus $\mathrm{E} 33 \mathrm{~L}$ & $>64$ & 0.67 & $18.7 \pm 2.0^{*}$ \\
\hline B. cereus ATCC 4342 & $>64$ & 0.17 & $20.6 \pm 5.1$ \\
\hline B. cereus ATCC 4342 & $>64$ & 0.67 & $21.6 \pm 2.6$ \\
\hline
\end{tabular}

${ }^{a}$ Minimum inhibitory concentrations (MIC) for PZN were determined by the microbroth dilution method and are reported as $\mu \mathrm{g} / \mathrm{mL}$

$b_{\text {The }} \mathrm{NaCl}$ concentration of the media is given in $\mathrm{M}(0.17 \mathrm{M}$ is the standard for $\mathrm{LB})$

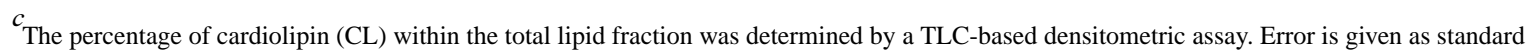
deviation with $n=3$. Asterisks indicate $\mathrm{P}<0.05$ relative to the same strain grown in $\mathrm{LB}$ with $0.17 \mathrm{M} \mathrm{NaCl}$. $\mathrm{PZN}$ was absent from CL quantification experiments 